\title{
Review Article \\ Reconfigurable and Tunable Metamaterials: A Review of the Theory and Applications
}

\author{
Jeremiah P. Turpin, Jeremy A. Bossard, Kenneth L. Morgan, Douglas H. Werner, and \\ Pingjuan L. Werner
}

Department of Electrical Engineering, The Pennsylvania State University, University Park, PA 16802, USA

Correspondence should be addressed to Douglas H. Werner; dhw@psu.edu

Received 15 November 2013; Accepted 7 January 2014; Published 22 May 2014

Academic Editor: Giacomo Oliveri

Copyright (C) 2014 Jeremiah P. Turpin et al. This is an open access article distributed under the Creative Commons Attribution License, which permits unrestricted use, distribution, and reproduction in any medium, provided the original work is properly cited.

\begin{abstract}
Metamaterials are being applied to the development and construction of many new devices throughout the electromagnetic spectrum. Limitations posed by the metamaterial operational bandwidth and losses can be effectively mitigated through the incorporation of tunable elements into the metamaterial devices. There are a wide range of approaches that have been advanced in the literature for adding reconfiguration to metamaterial devices all the way from the RF through the optical regimes, but some techniques are useful only for certain wavelength bands. A range of tuning techniques span from active circuit elements introduced into the resonant conductive metamaterial geometries to constituent materials that change electromagnetic properties under specific environmental stimuli. This paper presents a survey of the development of reconfigurable and tunable metamaterial technology as well as of the applications where such capabilities are valuable.
\end{abstract}

\section{Introduction}

Modern trends in technological development have increased demands for multifunctional components across the spectrum. In the radio frequency (RF) regime, wireless communications necessitate efficient, reconfigurable, tunable, inexpensive, and electrically small antennas that can be implemented in increasingly space-limited devices. In the terahertz band, many materials do not respond to in-band radiation and the components required to construct complex systems of terahertz devices, such as lenses, switches, and modulators, do not exist. Significant efforts are going into filling this "gap" in the spectrum [1]. Additionally, the emerging use of transformation electromagnetics/optics, particularly with regard to cloaking, requires spatial gradients that natural materials do not possess. With each of these challenges, designers must compromise among size, functionality, complexity, and fabrication cost.

Artificially constructed materials, metamaterials, have emerged as an attractive option for addressing many of these issues and have become a useful tool in optics and electromagnetics for the construction of devices with complex spatial- or frequency-domain behavior $[2,3]$. Recent developments in reconfigurable and tunable metamaterials have extended the possibility for fabricating metadevices [4] and unique, subwavelength devices with practical functionality. In addition to exhibiting electromagnetic responses not readily available in nature, these metadevices offer the possibility for improved performance characteristics in smaller, multifunction applications.

Strictly speaking, metamaterials are collections of far-subwavelength $(<\lambda / 10)$ resonating structures, typically aligned in a regular crystal lattice, and may be characterized as possessing either effective material parameters for bulk, 3D structures, or effective surface impedances for planar, 2D structures [5]. These resonators are designed to couple and interact with the free-space propagating electromagnetic waves, rather than be excited directly by a waveguide or transmission line. Metamaterials may be designed to yield a desired refractive index and intrinsic impedance or permittivity and permeability profile or to match a desired frequency-dependent scattering response, which may be 
viewed as a dispersive constraint on the effective material parameters. In practice, the term "metamaterial" is applied to any subwavelength resonator, whether in a collection or as an individual structure.

Many challenges have impeded the implementation of metamaterial-based devices, including the bandwidth limitations of strongly resonant devices, as well as fabrication limitations and tolerances. Many solutions to these problems have been presented which involve changes to metamaterial structure, composition, and constituent material properties. Tunable or reconfigurable metamaterials have great potential to alleviate many of the complications with passive metamaterials at the cost of increasing fabrication complexity and expense. Within this review, the definition of a tunable metamaterial is taken to mean a structure whose electromagnetic behavior is intentionally modified as part of the ordinary operation of the device through the influence of a change in, for example, the unit cell effective circuit, constituent material properties, or geometry. In addition to the conventionally defined free-space metamaterials, we also consider related structures including electromagnetic bandgap (EBG) materials or high-impedance surfaces (HIS), since they may be designed to mimic the properties of an effective magnetic conductor $[6,7]$.

This paper is intended to provide a comprehensive survey of published metamaterial tuning methods over all frequency bands and to give comparison information between competing techniques and their applications.

\section{Overview of Efforts and Techniques}

2.1. Metamaterials and Metamaterial-Inspired Devices. Many antenna [8, 9], microstrip/transmission line [10], and frequency-selective surface (FSS) applications use the term "metamaterial-inspired" to describe their use of resonant structures as loading or filter elements within the design, especially when the resonator in question has been repurposed from a metamaterial structure. The design and measurement procedure and goals may be viewed as a loose discriminator between a "metamaterial-enabled" and a "metamaterial-inspired" device. Metamaterial-enabled devices make use of the effective bulk or scattering properties of a metamaterial and will typically employ these desired effective properties in simulations during the design process. A metamaterial-inspired device, however, will rely on the exact behavior of individual resonators and will generally not utilize the metamaterial unit cells so as to obtain an effective bulk behavior. Although these structures are not true metamaterials, the fabrication approaches and design decisions share many similarities with metamaterial design, especially in the case of reconfigurable devices. Many of the examples in the literature of tunable metamaterials are more properly metamaterial-inspired devices which use individual resonators (most commonly a split-ring resonator (SRR)), often strongly coupled to transmission lines or antennas, as the proof of concept or even the final application for the design. We include these papers in this review since the challenges towards implementation are shared for both approaches and a reconfigurable metamaterial-inspired transmission line or antenna may be viewed as the first step in the development of bulk tunable metamaterial-enabled devices.

2.2. Tuning Method versus Frequency. A variety of tuning methods have been examined in the literature to generate dynamic changes in a metamaterial's performance. These include direct changes to the unit cell's circuit model by varying capacitance or conductance, using electrically, chemically, thermally, or optically sensitive materials to change the constituent material properties of a structure and therefore change its electrical response and altering the geometry of the unit cell through stretching, shifting, or deforming all or part of the structure. Some of these techniques (such as varactor diodes) have been applied for operation at particular wavelengths, while others (such as phase-change materials) have been applied across the electromagnetic spectrum. Each of these tuning methods is described in more detail in Section 3. Figure 1 shows a diagram of tuning methods versus operational frequency for all reconfigurable metamaterial and metamaterial-inspired devices reviewed in this paper.

2.3. Common Unit Cell Designs. The base unit cell on which a reconfigurable metamaterial device is designed determines the fundamental behavior of the structure. In most cases, the starting point for the reconfigurable device is a static design which is then augmented with the tunable component, material, or structure. The physical geometry of the unit cell determines the electromagnetic coupling into the metamaterial, provides (potentially desirable or undesirable) frequency selectivity, and can allow for cancellation of electric (in the case of the SRR) or magnetic (for the electric LC resonator) excitations as well as for strongly coupling the electric and magnetic responses. Especially at optical wavelengths, many unit cells do not explicitly couple to the electric or magnetic fields but are tuned to resonate simultaneously in the same geometry [11]. Unit cells with well-defined resonant and EM-coupling properties are commonly used to implement metamaterials with desired material properties, while designs for scattering control or antenna enhancements may be less sensitive to cross-polarization or E-H field (magneto-electric) coupling. Most tunable metamaterials are based on common elemental building blocks or particles, such as the widely used SRR $[12,13]$, the complementary split-ring resonator (CSRR) [14-16], and their electric-field coupled cousin the electric LC resonator (ELC) [17-21]. Often, the structure is modified to a greater or lesser degree to accommodate the constraints of the tuning mechanism $[22,23]$. Tunable impedance surfaces are generally constructed from grounded square patches (mushroom-type artificial magnetic conductor (AMC) structures) [24-33]. Although basing a design on an existing resonator can be useful, many studies have developed new structures subject to limitations or to specifically leverage the capabilities of a given tuning mechanism; this includes the use of reorientable or variable distributions of colloidal nanoparticles [34-38], interconnected grid structures [39-41], and optimized binary FSS patterns [42, 43]. Transmission-line 


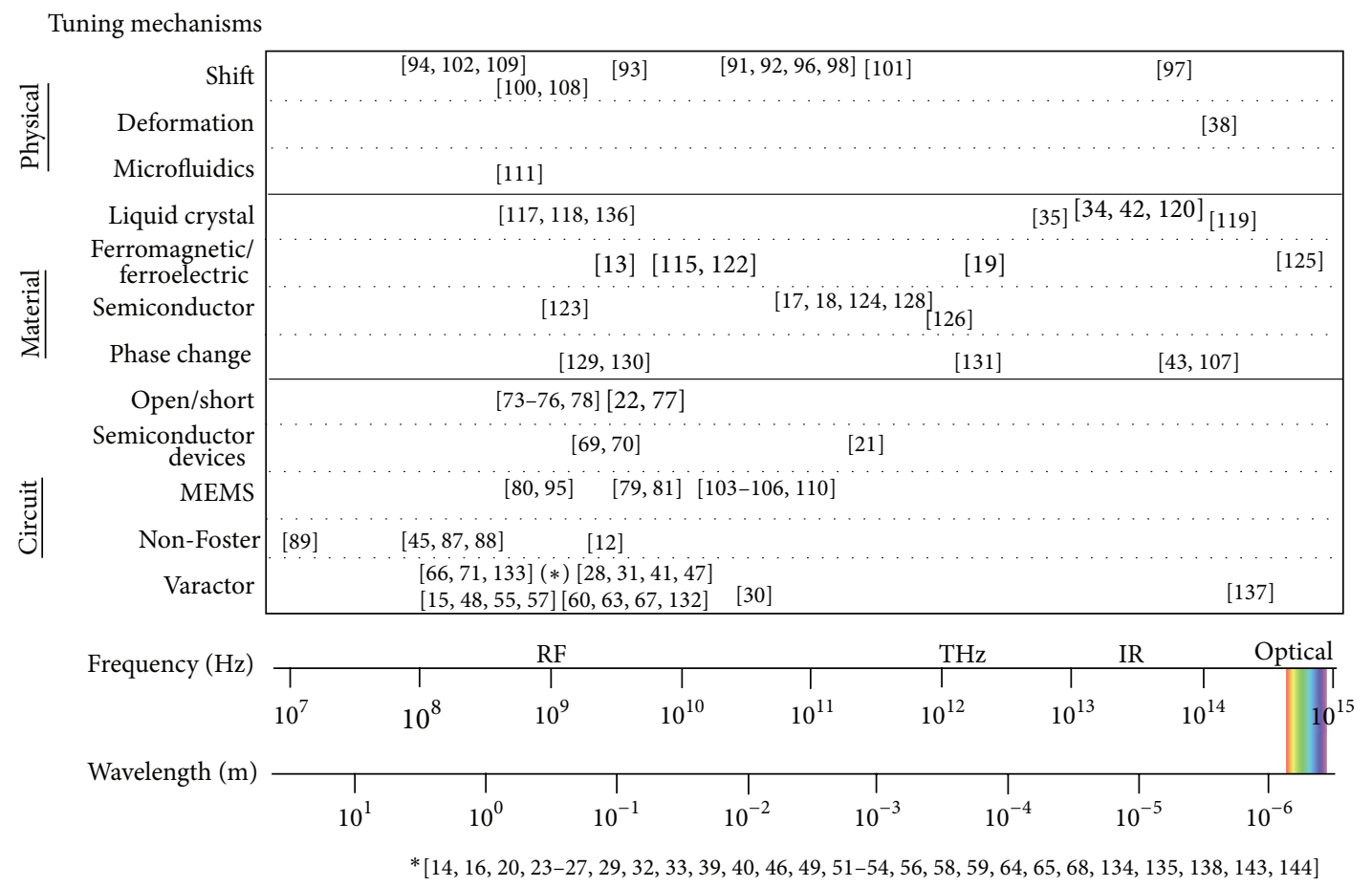

FIGURE 1: Examples of metamaterial tuning mechanisms in the literature plotted against their operational frequency. Groups of references are centered on their shared operational frequency.

metamaterials may not use such a recognizable geometric unit cell pattern as an SRR or spiral but achieve periodic behavior instead based on their equivalent circuit model [44-46].

\section{Tuning Mechanisms}

The various tuning mechanisms that have been demonstrated in the literature may be divided into three general categories. Circuit tuning involves the targeted insertion or modification of individual impedances into the unit cell circuit model; this includes the use of variable capacitors and switches within and between unit cells. We classify geometric tuning as those methods that physically perturb the structure of the unit cell in such a way that many or multiple changes occur in the equivalent circuit model. This would include moving subsets of the unit cell relative to a fixed point or using MEMS devices to change orientation or location of a significant fraction of the unit cell. Finally, material tuning leverages changes in material parameters of a substrate, patterned layer, or small region of the unit cell to modify the response and properties. Phase change materials and liquid crystal devices are examples of this technique. The remainder of this section is devoted to describing the use of these methods as reported in the literature.

3.1. Circuit Tuning. The electromagnetic behavior of real, passive transmission lines, antennas, and metamaterials may be represented as an equivalent circuit composed of lumped inductive, capacitive, and resistive elements. This decomposition of complex geometric structures into a well-defined circuit model is highly useful for predicting the response of modified or perturbed designs. SRRs, for example, may be represented as a parallel LC or RLC tank circuit (possibly with additional parasitic elements depending on the frequency and exact geometry) where the inductive element is coupled to the incident field. We classify circuit tuning of metamaterials as those methods which insert, modify, and control individual elements in the metamaterial's equivalent circuit.

3.1.1. Varactor Tuning. Varactor diodes represent the single most popular tuning technique due to their simple integration into many kinds of metamaterials. By acting as a voltage-controlled capacitance, varactors are convenient for controlling the resonant frequency of SRRs or other types of resonant unit cells. Limitations to their universal application include losses due to nonzero series RF resistance, reduced performance at higher frequencies, and challenges with distributing the required DC bias signals throughout a metamaterial. Varactors are very good approximations to ideal tunable capacitors at $\mathrm{MHz}$ frequencies, but significant parasitic impedances accumulate and limit many metamaterial applications above $4-10 \mathrm{GHz}$. Bias distribution has been implemented for several specific metamaterial design styles. Using varactors to reconfigure planar metamaterials (such as HIS or AMC) alleviates the bias distribution issue by allowing bias signals to be delivered through the vias and traces behind the ground plane, isolated from the incident RF signals. 
Applying varactors to transmission-line metamaterials also simplifies the bias distribution [14-16, 23, 47-51], since additional traces to provide bias levels can be easily sited to avoid distortion of the desired frequency response. Bulk $3 \mathrm{D}$ metamaterials or transmissive planar metamaterials are more challenging, but several implementations have showed success by basing the geometry on an interconnected grid, potentially with lumped resistance or inductance to prevent RF coupling [52]. Figure 2 shows an example of a tunable AMC structure with integrated bias lines.

Varactors are used to augment capacitive coupling within metamaterial structures, for example, across an existing capacitive gap [20,31,54-60] or between adjacent unit cells $[24,26,27,29,32,33,39-41,61]$, as well as to completely change the equivalent circuit by introducing a tunable capacitance in place of an inductor, open, or short, as done in combined right-left handed (CRLH) transmission line metamaterials $[62,63]$. The former, by tuning the values in an essentially unchanged equivalent circuit, adjusts the resonant frequency of the device, without the dramatic change in behavior seen in the latter case.

As a semiconductor device, use of a varactor in a metamaterial design is occasionally referred to as an "active" metamaterial $[33,53,54,64,65]$ although the device remains passive for RF frequencies and is only active in its requirement for a DC bias. Most examples have, with reasonable accuracy, treated the varactors in their designs as ideal or nearly ideal linear capacitors, but several studies have examined in more detail the nonlinear effects of the varactor at low and high power levels $[55,56]$.

Some initial descriptions of new tunable metamaterials have used explicit lumped capacitors with different discrete values to approximate the varactor capacitance tuning behavior and demonstrate the desired tuning effects [6668]. This strategy represents a first step to a full, tunable implementation of the metamaterial.

The use of PIN diodes [69-71] and digital potentiometers [72] in a metamaterial shares similarities with the use of varactors in terms of their application and ease of integration, but these actuators affect resistance rather than capacitance. The advantages of simple control and liabilities of control signal distribution and frequency limitations are also comparable to varactors. By tuning resistance, these methods could be applied to control or augment losses in a metamaterial absorber design.

3.1.2. (Nearly) Ideal Switches. An ideal RF switch would be capable of instantly changing states from a $0 \mathrm{Ohm}$ perfect zero-electrical-length connection to a perfect open circuit. Since this device does not exist, several approximations to this ideal have been applied for the design of reconfigurable metamaterials.

The simplest approximation of an ideal switch is to replace the prototype containing a switch by a pair of prototypes or simulations, one with a metallic short and the other an open circuit at the desired location of the switch [22, 73-78]. This technique is simple and can be useful to provide a proofof-concept or intermediate design demonstration when no actuator exists that can satisfy the design requirements for a given structure.

Micro-electro-mechanical systems (MEMS) can be used to fabricate RF switches with very high efficiency. Here, we consider the use of MEMS as localized (relatively) selfcontained switches between two points in the metamaterial equivalent circuit, rather than tuning by changing the geometry of the unit cell (which is discussed in Section 3.2). Applying multiple switches per unit cell or group of unit cells allows multiple responses to be generated, as illustrated in Figure 3. MEMS switches that physically make and break contact between two terminals can be manufactured, but most RF MEMS switches are better analyzed as high-magnitude capacitance modulators. In electromagnetics terms, tuning between a large and a small capacitance will yield a good approximation to an ideal RF switch. Unlike varactor diodes, the capacitance ranges achieved by MEMS actuation can often be sufficient to yield nearly ideal switching behavior and are used as more realistic alternatives to open/short actuation [22].

MEMS switching performance is exceptional [79-81]. Limitations on the use of MEMS switches include high actuation voltages (on the order of 70-150 V) that are difficult to interface with CMOS control circuits, high manufacturing variability in the "off" -state capacitance between individual devices [80], and either complex fabrication requirements for an integrated design or expensive per-unit costs for the purchase of commercially packaged devices. For these reasons, the use of MEMS as RF switches in metamaterials has been limited, with published examples demonstrating either a single or a small number of unit cells.

3.1.3. Active, Non-Foster Metamaterials. Non-Foster refers to active circuitry that is not subject to Foster's reactance theorem [82-84] which states that the reactance slope versus frequency for any real, passive circuit must be nonnegative. Non-Foster circuits, then, through the use of active circuitry in the form of a negative impedance converter $[85,86]$, can achieve a negative impedance slope. This unique characteristic allows true broadband impedance manipulation through complete or partial cancellation of reactance dispersion over some bandwidth, rather than only at a point as is possible with combinations of inductive and capacitive loads. Partial cancellation of reactance dispersion in a metamaterial through the use of a negative inductance or capacitance may be used to maintain a "resonant" effect over a wide operation band [62].

Applying a non-Foster load to a metamaterial or AMC structure $[83,87]$ to broaden a resonance band can be viewed as a type of frequency-selective tuning [12], making such devices $[45,62,88,89]$ a close cousin to other active [21] and tunable metamaterials. Both share many of the same design considerations as to the inclusion of active devices, control, and bias circuitry into the limited physical space of a metamaterial unit cell. Future work that includes tunable or reconfigurable non-Foster element may yield many advantages in terms of bandwidth and shared complexity. Figure 4 demonstrates the dramatically increased AMC bandwidth 


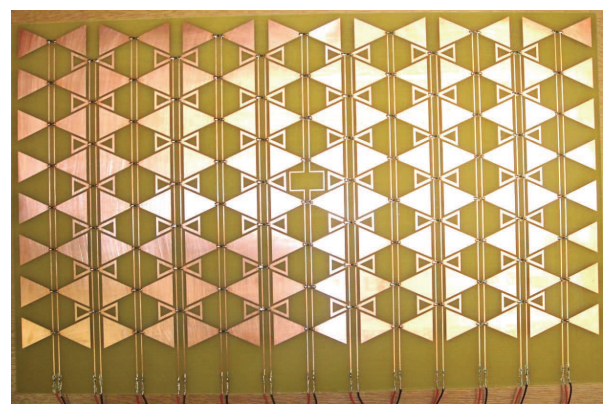

(a)

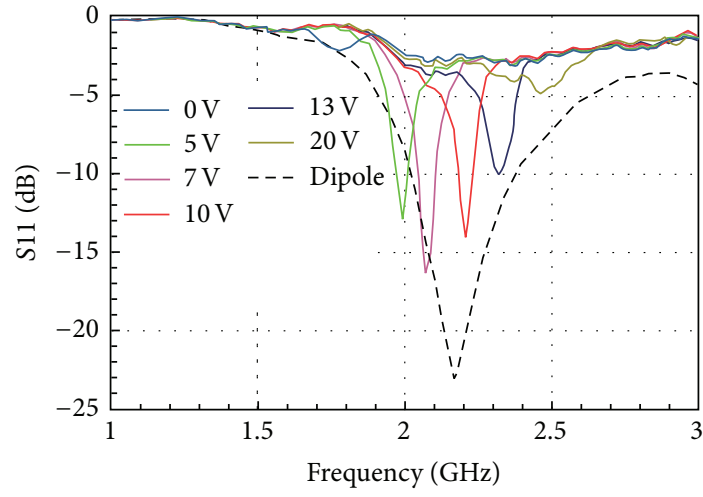

(b)

Figure 2: (a) Bow-tie AMC structure with bias lines to varactor diodes running throughout the structure. (b) When placed beneath a dipole antenna, tuning the varactors in the AMC controls the resonant frequency of the dipole. Used by permission [53].

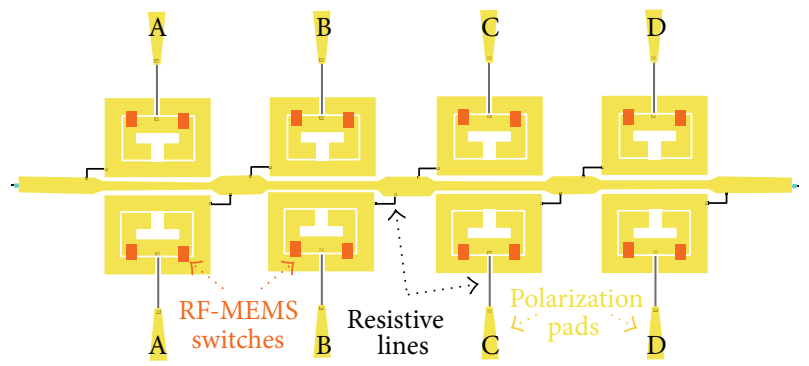

(a)

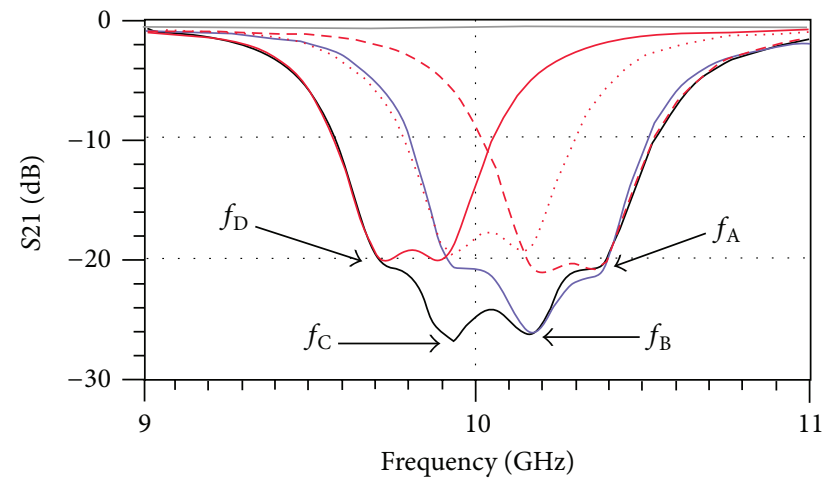

(b)

FIGURE 3: (a) The SRR-loaded microstrip line with RF-MEMS switches controlling the resonances acts as a bandstop filter. (b) The stopband frequency can be altered by changing the combination of switch states. Used with permission [79].

that can be achieved with non-Foster loads over a similar AMC implemented with varactor loading.

As with all active circuits, individual non-Foster devices must be frequency-limited to preserve stability. The examples of non-Foster metamaterials have been implemented primarily in the $\mathrm{MHz}$ range for size reasons as well as amplifier stability limitations.

3.2. Geometrical Tuning. Many metamaterials rely on conducting elements that can couple with impinging electromagnetic waves in order to achieve a desired electric or magnetic resonance or other effective behavior. Because the metamaterial properties typically depend on the shape, size, orientation, and proximity of the conducting elements, techniques that alter the geometry of the conducting elements can provide an excellent means for tuning or switching the metamaterial response.

At optical wavelengths, one nascent technique for geometrical tuning is to use self-assembly to align resonant nanorods in solution [36, 37]. In this method the entire conducting elements are aligned by applying a local electric field gradient via electrodes. The gold $(\mathrm{Au})$ nanorods have a different resonant wavelength depending on whether the incident electromagnetic wave is aligned with the electric field oriented along the length of the rod or orthogonal to the length. Hence, the metamaterial response can be changed by aligning the nanorods in different orientations or by allowing them to disperse in the solution. Another related technique disperses Au nanorods in liquid crystal or other anisotropic fluids, so that the Au nanorods can be aligned with the liquid crystal molecules [90]. This technique helps to align the conducting nanorods within a larger volume and points toward a capability of realizing bulk reconfigurable metamaterials.

Another related geometry tuning technique that has been demonstrated in the $\mathrm{THz}$ regime is tilting the resonant conducting elements in order to alter the metamaterial response [91, 92]. In both of these demonstrations, SRR elements were tilted using MEMS [91] or thermal [92] control. The SRR element is anisotropic in both effective permittivity and effective permeability, so tilting the element causes it to couple differently with an incident wave. Hence, tilting techniques are useful for reconfiguring the absorption or filtering spectrum of the metamaterial as well as causing electric or magnetic resonances to appear and disappear in regions of the electromagnetic spectrum.

In addition to reorienting entire elements, metamaterials can be geometrically tuned by moving conducting elements in relation to each other [38, 81, 93-108]. MEMS are frequently employed to perform the mechanical movement of conducting elements for $\mathrm{THz}$ metamaterials [38, 80, 93, 95, 101]. When conducting elements are moved closer or further 


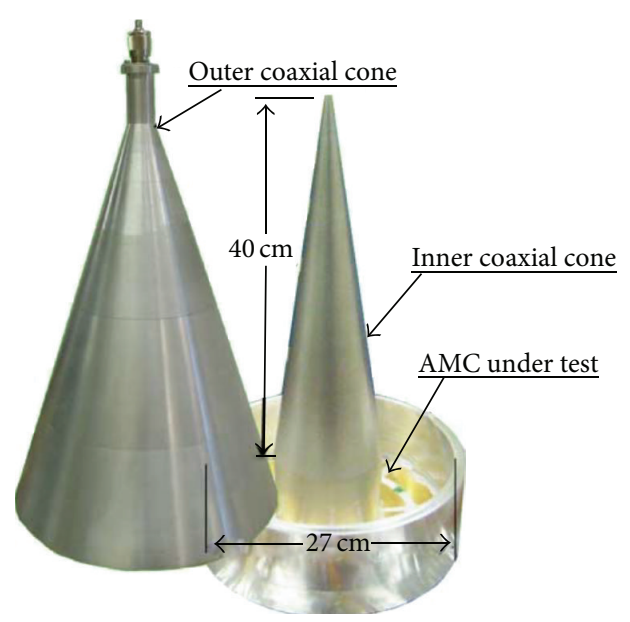

(a)

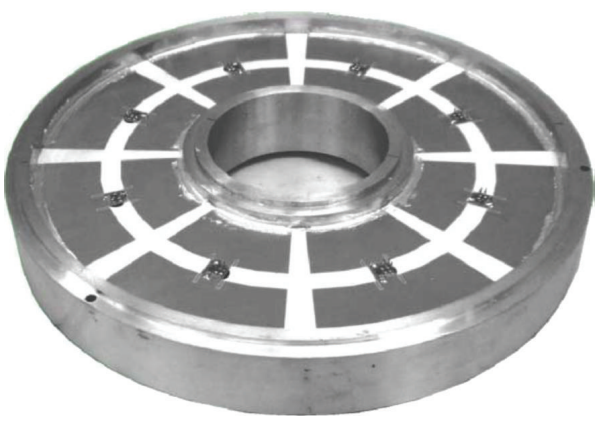

(b)

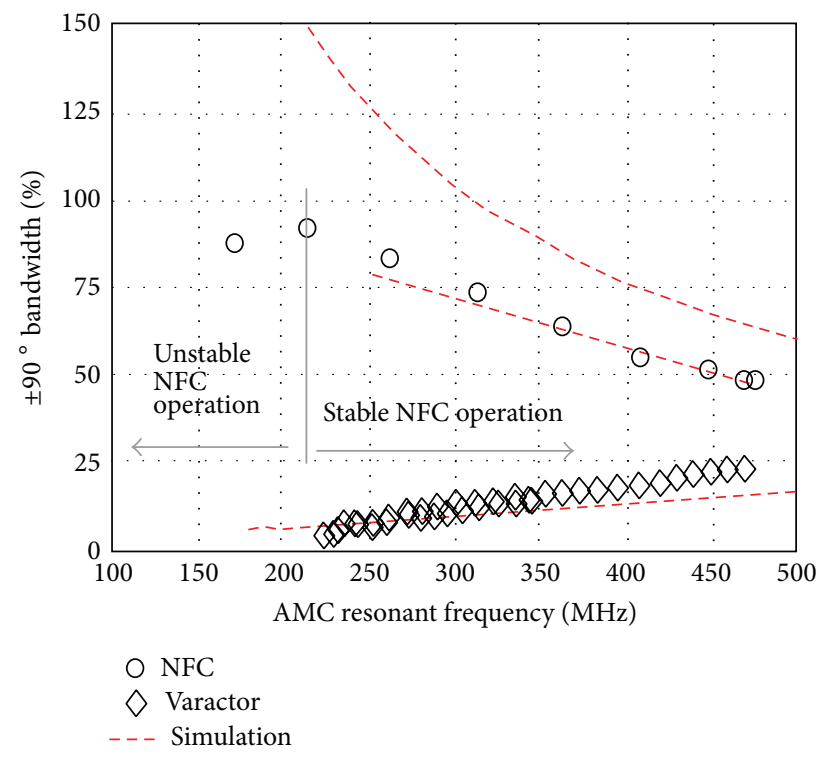

(c)

FIgURE 4: (a)-(b) Coaxial TEM reflection test cell for cylindrical non-Foster circuit (NFC) negative-impedance-loaded AMC structure. (c) The non-Foster-loaded AMC shows greatly increased bandwidth compared to a similar varactor-loaded AMC design. Used by permission [87].

apart, the coupling between them changes, which can result in shifts in resonance frequency or changes in resonance strength. Moving conducting elements can also reconfigure the shape of the element. For example, in [101] one arm of a cross can be disconnected and moved to reconnect with the neighboring cross element. This kind of reconfiguration changes the response of the metamaterial from being polarization independent to being dichroic. One novel technique for moving conducting elements in a metamaterial involves stretching the substrate, so that the elements on the substrate become further separated [97], producing large shifts in the resonant frequency of the elements. Several tunable HIS have been demonstrated that operate by mechanically shifting an upper plate of elements along the surface [102, 109] or vertically [105] in order to adjust the phase of the reflected wave, illustrated in Figure 5. Tuning the phase of a HIS is effective for steering a radiated beam from an antenna system $[102,109]$. Several tunable HIS have also been demonstrated that employ MEMS to raise or lower capacitive conducting plates in the unit cell $[103,104,106,110]$. This technique effectively changes the capacitance in a part of the element and operates similar to the circuit tuning techniques described in Section 3.1.

Perhaps the most impressive form of geometric tuning is described in [111], where mercury (Hg) SRRs were formed by using microfluidics to inject $\mathrm{Hg}$ into SRR molds to form the resonators. This system can place the $\mathrm{Hg}$ resonators and remove them in real time in order to reconfigure the metamaterial response.

Geometrical tuning can provide drastic changes in the metamaterial properties because the shape of the conducting elements has such a large influence on the corresponding resonance. However, implementing geometrical tuning is challenging because a physical control mechanism is needed 

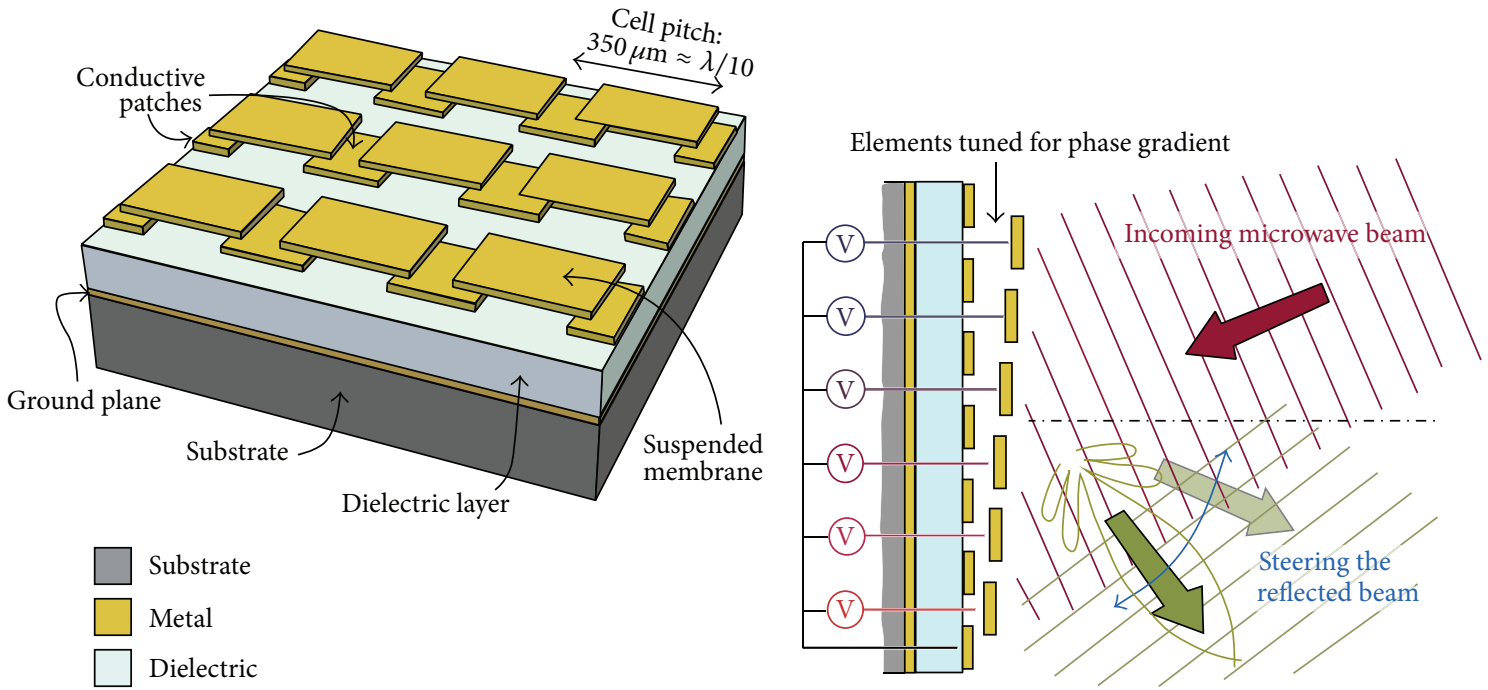

(a)

(b)

FiguRE 5: (a) Vertical motion of a suspended conductive membrane changes the resonant frequency of the high-impedance surface. (b) Varying the MEMs actuation voltage across the surface allows the metamaterial to act as a reflectarray. Used by permission [105].

to manipulate the conducting elements. Typically, the control mechanisms, whether they are electrodes for self-assembly or MEMS actuators, are complicated to design and fabricate and they need to be accounted for in simulation.

3.3. Material Tuning. While changing the shape of resonant elements provides a range of opportunities for tuning, the constituent materials that make up the unit cell ultimately control the properties of the metamaterial. In the literature, a variety of constituent materials have been evaluated and exploited for tuning metamaterials by controlling the permittivity, permeability, and conductivity of parts of the unit cell.

The electrical size of the conducting resonant elements is affected by the permittivity of the surrounding medium. For instance, a dipole element in free space is resonant when its length is approximately half of the wavelength. However, if this dipole is embedded in a dielectric, it becomes resonant at half the wavelength size in the dielectric. This means that if tunable dielectric materials can be incorporated in the unit cell, the resonance wavelength of the metamaterial can be tuned as the constituent material permittivity changes. Several papers have theoretically investigated the effects of changing the substrate permittivity on a metamaterial response [112-114]. There are a few candidate materials that have been used for permittivity tuning of metamaterials, including $\mathrm{Ba}_{0.5} \mathrm{Sr}_{0.5} \mathrm{TiO}_{3}$ (BST) ferroelectric films, liquid crystal, and Ga-Sb-Te (GST) phase-change materials. BST films provide a permittivity change under voltage bias and have been used as a substrate for tuning SRR elements along a transmission line at microwave frequencies $[115,116]$. BST can also be tuned via change in temperature as demonstrated in [13] for tuning the resonance frequency of SRRs. Liquid crystals offer dielectric tuning over a broad range of the electromagnetic spectra from RF all the way through optical wavelengths. Liquid crystals are anisotropic in permittivity due to their composition of long, aligned molecules and exhibit changes in permittivity due to applied voltage, induced magnetic field, optical excitation, and even thermal change, which can be exploited to tune metamaterials. There are a number of theoretical and practical demonstrations showing the tuning of negative index materials and magnetic resonances at optical wavelengths $[34,35,42,117-120]$, including the example shown in Figure 5. The same shift in magnetic resonance has been explored for designing a metamaterial that switches between being highly transmissive and reflective by reorienting liquid crystal molecules included in the unit cell [119]. GST phase-change materials have discrete crystalline and amorphous phases that possess distinct electrical properties at infrared wavelengths and that can be switched between by subsequently melting and cooling the material. Several papers have investigated the use of GST as a constituent material for tuning metamaterials [43, $107,121]$. GST was used as the resonant element in [43] to change scattering behavior among being highly reflective, transmissive, or absorptive. GST was also explored as a substrate in $[107,121]$ that can switch a metamaterial mirror between reflection polarization states.

Permeability tuning can also be achieved at RF using ferrite materials. In [122], yttrium iron garnet (YIG) rods having a negative permeability are incorporated into a metamaterial unit cell. Applying a magnetic field bias allows the permeability of the YIG rods to be tuned from negative to positive, causing a change in response of the metamaterial. While negative permeability constituent materials are not available at $\mathrm{THz}$ or higher frequencies, ferrite materials can be a useful tuning method at RF.

The third general area for constituent material tuning is conductivity. Conductivity change is frequently accomplished using semiconductor materials with applied voltage 
[17] or optical pumping [124-128]. The semiconductor can be incorporated as a substrate under all elements $[17,128]$ or as an inductive load over part of the resonant elements [125-127] to tune their resonant frequency. Graphene has also been used to inductively load resonant elements in a HIS when under a voltage bias [129]. A variety of techniques have also been employed to change the conductivity of the resonant elements themselves. Semiconductor SRRs have been used with a change in conductivity achieved thermally [18] or by applying a magnetostatic field [19]. Conducting polymers have also been used as resonant elements in metamaterial absorbers [75]. The conducting polymers exhibit large changes in conductivity (i.e., from resistive to conductive) when stimulated by certain chemical analytes. This enables the metamaterial to reconfigure from reflecting to absorbing or to change resonant frequencies. $\mathrm{VO}_{2}$ phasechange materials also exhibit large conductivity changes with a voltage bias and have been used to form the resonant elements in several metamaterial experiments [130-132].

Bulk material-based tuning is ultimately limited by the range of electromagnetic responses available in the constituent substances, where each material system poses unique implementation challenges. For instance, BST and $\mathrm{VO}_{2}$ offer useful permittivity and conductivity changes, respectfully, but they are both sensitive to temperature and thus must be used in temperature controlled environments. GST phasechange material on the other hand does not suffer from temperature variation, but incorporating the heating and cooling mechanisms into the metamaterial to control the phase transitions between crystalline and amorphous states is challenging. Research needs to continue in the development of tunable material systems that can be harnessed to meet application specific reconfigurable metamaterial needs.

\section{Applications and Tuning Goals}

The inherent nature of tunable and reconfigurable metamaterials affords them the ability to be used in a wide variety of applications. The distinctions between these end goals are largely a matter of perspective. Shifting the frequency of a passband is functionally identical to changing from a transmissive to reflective surface at a fixed frequency. Interpretation of an identical change in response can be made in terms of scattering parameters, effective material parameters, or the metamaterial's loading effect on an adjacent antenna or transmission line. We acknowledge the discretionary nature of categorizing tunable metamaterials into intended applications; however, in this literature survey, we define four broad common application areas. First, we discuss the design of tunable metamaterials as an enabling technology for tunable filters and antennas; this focuses on metamaterials that are primarily designed to shift the resonant frequency or alter the device's bandwidth. We then note metamaterials that are optimized to modulate the scattering (transmission, reflection, and absorption) of a material at a given resonant frequency. We classify designs meant to spatially vary the index of refraction with a focus on transformation electromagnetics. Finally, we recognize tunable structures that manipulate wave propagation characteristics including directivity, radiation pattern, polarization, and propagation mode.

4.1. Tunable Filters and Antennas. A major focus of tunable and reconfigurable metamaterials lies with designing tunable filters and frequency-reconfigurable antennas. One desirable feature of such devices is tunable frequency band selectivity. In wireless communications, using tunable metamaterials enables wider ranges of operating bands within a minimal geometry. Bossard et. al. have applied liquid crystal superstrates to shift the frequency of the transmission band in an infrared FSS device, as illustrated in Figure 6. Reynet and Acher demonstrated tunable varactor-loaded metamaterials based on conducting coils [57]. Later works introduced this concept into transmission lines and antennas, where tuning was achieved by varying capacitance, typically of SRRs and CSRRs [16, 23, 46-50, 62, 123, 124, 133]. Zhu et al. demonstrated an electrically small, tunable SRR antenna operating in the UHF frequency band [123]. As shown in Figure 7, this varactor-loaded dipole-like structure provides a tunable narrowband (notch filter) alternative to wideband antennas in RF communication systems. The antenna has the advantage of compact size, low cost, and easy fabrication as well as implementation. Researchers have attained similar RF frequency tunability through a variety of other methods, such as through MEMS [22, 73, 79, 81, 93, 94], tunable EBG surfaces [59, 78, 95, 112, 134, 135], ferroelectric rods and films $[13,115,116,122]$, vanadium dioxide switches [130, 131], and liquid crystals [42, 117, 118, 136]. These proposed designs exhibited varied tunable RF filter types, such as band-stop, band-pass, and notch filters. Whereas the filters discussed above are actively voltage-controlled, designers have also demonstrated nonlinear varactor-loaded (or p-i-nloaded) metamaterial filters that vary with incident power, making them ideal for nonlinear devices such as RF limiters $[55,56,69]$. Frequency tunability is especially desirable for fabricating flexible devices in the terahertz and optical bands. Such devices could include sensitive platforms for measuring chemical or biological agents, temperature sensors, and intelligent detectors among other applications [18, 19, 96, $97,125,126,137]$. Furthermore, in contrast to scanning the center frequency of a desired response feature, another essential functionality is tunable bandwidth, or reconfigurable filtering type, that is, dynamically switching between bandpass, band-stop, notch, and so forth. Several authors have demonstrated metamaterial structures that exhibit reconfigurable filter types, as well as tunable center frequencies $[14,39,63]$. Tunable filters, FSSs, and antennas have wide ranges of applications across the electromagnetic spectrum. Tunable metamaterials offer a cost-effective, compact, and flexible option for implementing tunable band selectivity and reconfigurable filter functionality in applications ranging from RF communication system design to optical sensors and detectors.

4.2. Scattering Parameter Tuning. Tuning the transmission, reflection, and absorption characteristics of a material at 


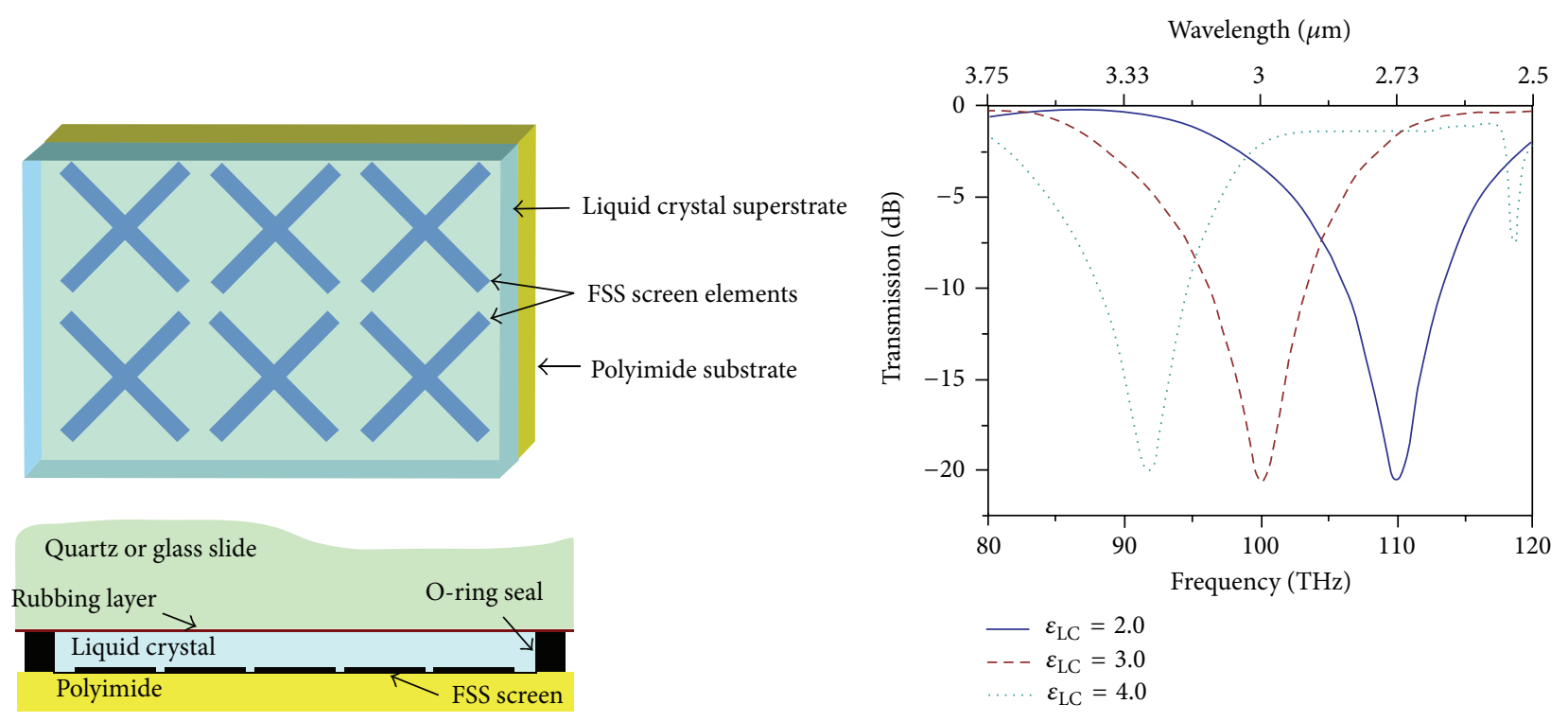

(a)

(b)

Figure 6: (a) An illustration of a liquid-crystal-based tunable FSS unit cell. (b) Changing the applied potential difference between the FSS and the quartz slide changes the liquid crystal orientation and the scattering response of the FSS. Used by permission [42].

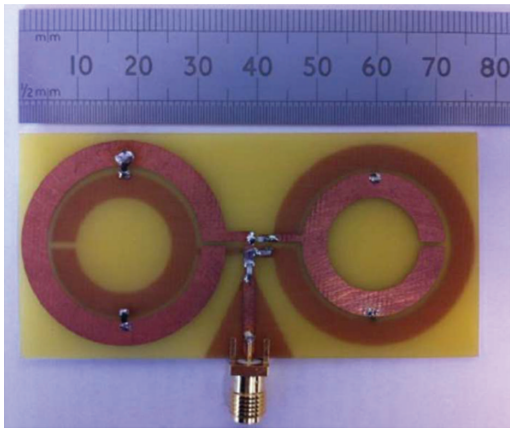

(a)

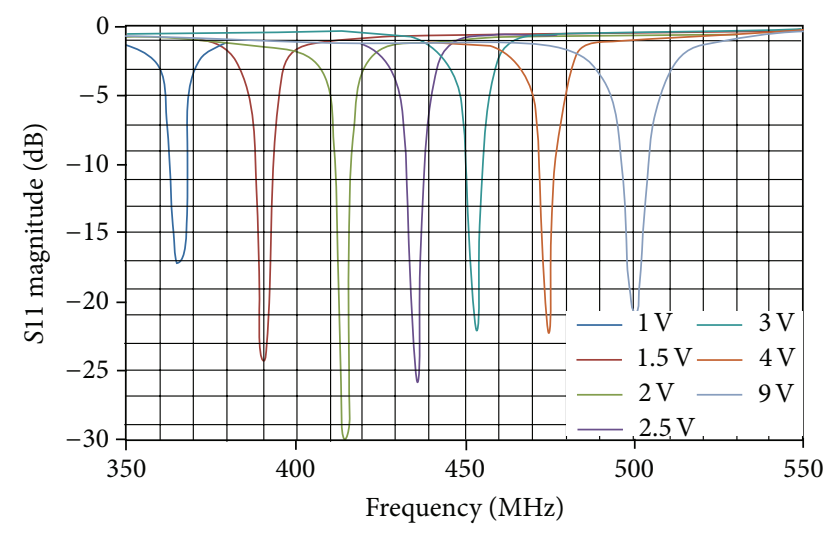

(b)

FIGURE 7: (a) This varactor-loaded dipole-like antenna has a (b) narrow-band but tunable notch-filter response, allowing operation over a wide range of frequencies while avoiding spurious signals. Used by permission [123].

a particular frequency affords the designer the creation of devices such as switches, modulators, FSSs, absorbers, sensors, and more. Tunable metamaterials offer avenues for realizing these devices in low-profile mediums with ultrafast tuning capabilities. A fundamental goal for dynamically controlling the scattering from a material is amplitude modulation. Tunable metamaterials enable designers to create high-speed modulators that alter the transmission and/or reflection amplitude of an incident electromagnetic wave [17, $21,67,111,127,138]$. Practical devices emerge from this fundamental functionality, such as high-speed switches operating in the terahertz and near-infrared bands $[38,98,128]$. Ou et al. demonstrated a switchable metamaterial that operates in the optical band at three orders of magnitude faster than previously achieved [38]. As shown in Figure 8, electrostatic forces were used to drive parallel strings at megahertz frequencies to modify the transmission and reflection spectra of the device. The characteristics of this device make it ideal for protective optical circuitry and reconfigurable optical networks. Tunable scattering characteristics are also vital to developing tunable cloaks and electromagnetically transparent materials. In particular, by controlling the reflection and transmission, designers can create perfect absorbers, reduce radar cross section, or achieve complete transmission and reflection from a surface $[29,68,70,75,91,92,119,139]$. Tao et al. designed a MEMS-based structurally tunable metamaterial that can dynamically modify its transmission amplitude in the terahertz regime [91]. Rotating the unit cell plane with respect to the incident electric and magnetic fields alters the coupling efficiency. Structural reconfiguration time can be 

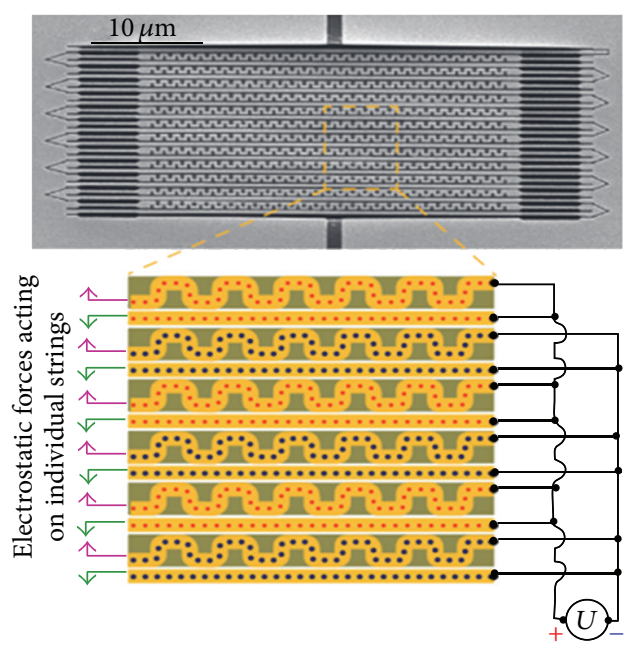

(a)

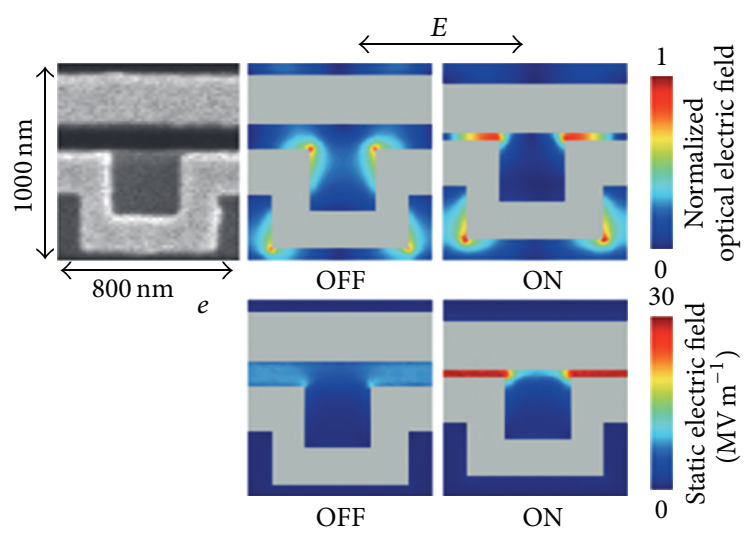

(c)

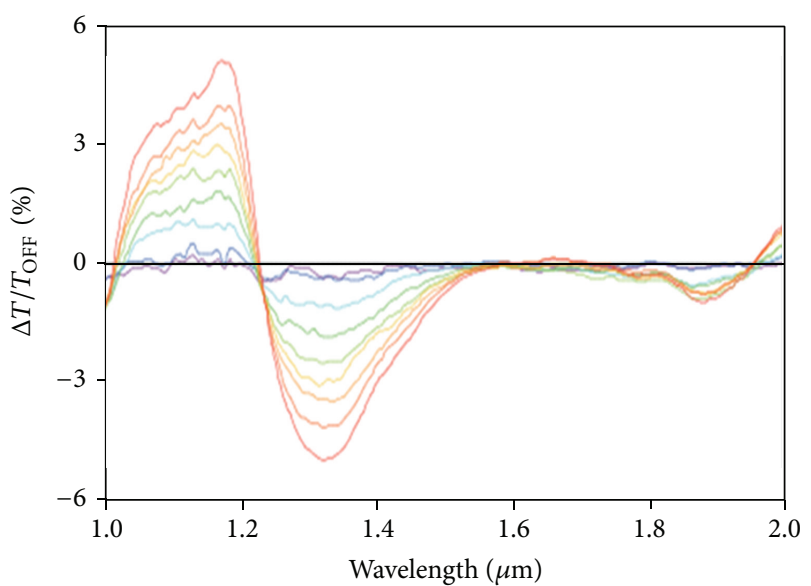

(b)

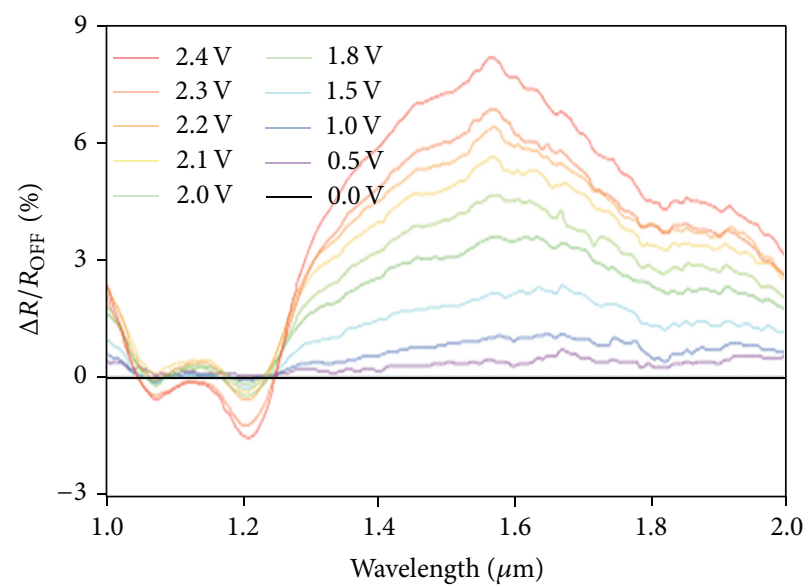

(d)

FIGURE 8: (a) Scanning electron microscope (SEM) image of photonic metamaterial device and schematic of driving circuit. (c) SEM image of single metamolecule and plasmonic field distribution for OFF and ON states. (b) Transmission and (d) reflection spectra of photonic metamaterial at varied induced static voltages. Used by permission [38].

on the order of milliseconds, allowing this design to be used in applications ranging from reconfigurable filters, thermal cantilever-based detection, or tunable absorbers, cloaks, or concentrators. Controlling scattering also has several applications in the optical band; varying transmission and reflection enables the development of optical temperature sensors, switches, modulators, and other planar optical metamaterialenabled devices $[90,99]$. One particular application is in compressive sensing. Werner et al. demonstrated a phase change material that could spatially modify its complex index of refraction to dynamically change the effective size and shape of an aperture [43]. This beam-forming aperture, with its ultrafast response in the infrared band, could then be used for compressive sensing, a technique where few information rich measures are sampled to construct highresolution images. This technique is a step towards achieving the desired resolution while maintaining the area coverage and minimizing the cost, size, weight, and power of the sensor system. Controlling the scattering characteristics of a material enables designers to create a wide variety of common devices across the electromagnetic spectrum.

4.3. Spatial Tuning for GRIN Lenses. The recent explosion of research in transformation electromagnetics techniques $[140,141]$ has increasingly put emphasis on spatially modifying the parameters of materials. In particular, these spatial gradients are primarily focused on altering index of refraction. Sheng and Varadan investigated the effect of substrate dielectric (relative permittivity) on a metamaterial structure [113]. By varying the relative permittivity from 1 to 14 , the structure's resonant frequency dropped from $16 \mathrm{GHz}$ to $6 \mathrm{GHz}$ [114]. This discovery provided a foundation for designing voltage tunable dielectric substrates, which led to the development of microfabricated artificial dielectrics for microwave circuits [100]. While this research addressed only modifying the overall index of refraction of a substrate, several authors developed methods for creating materials 


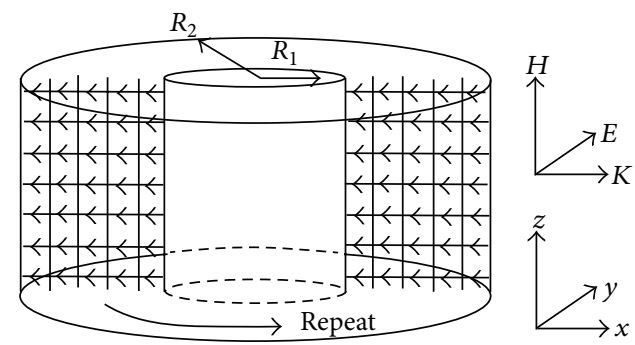

(a)
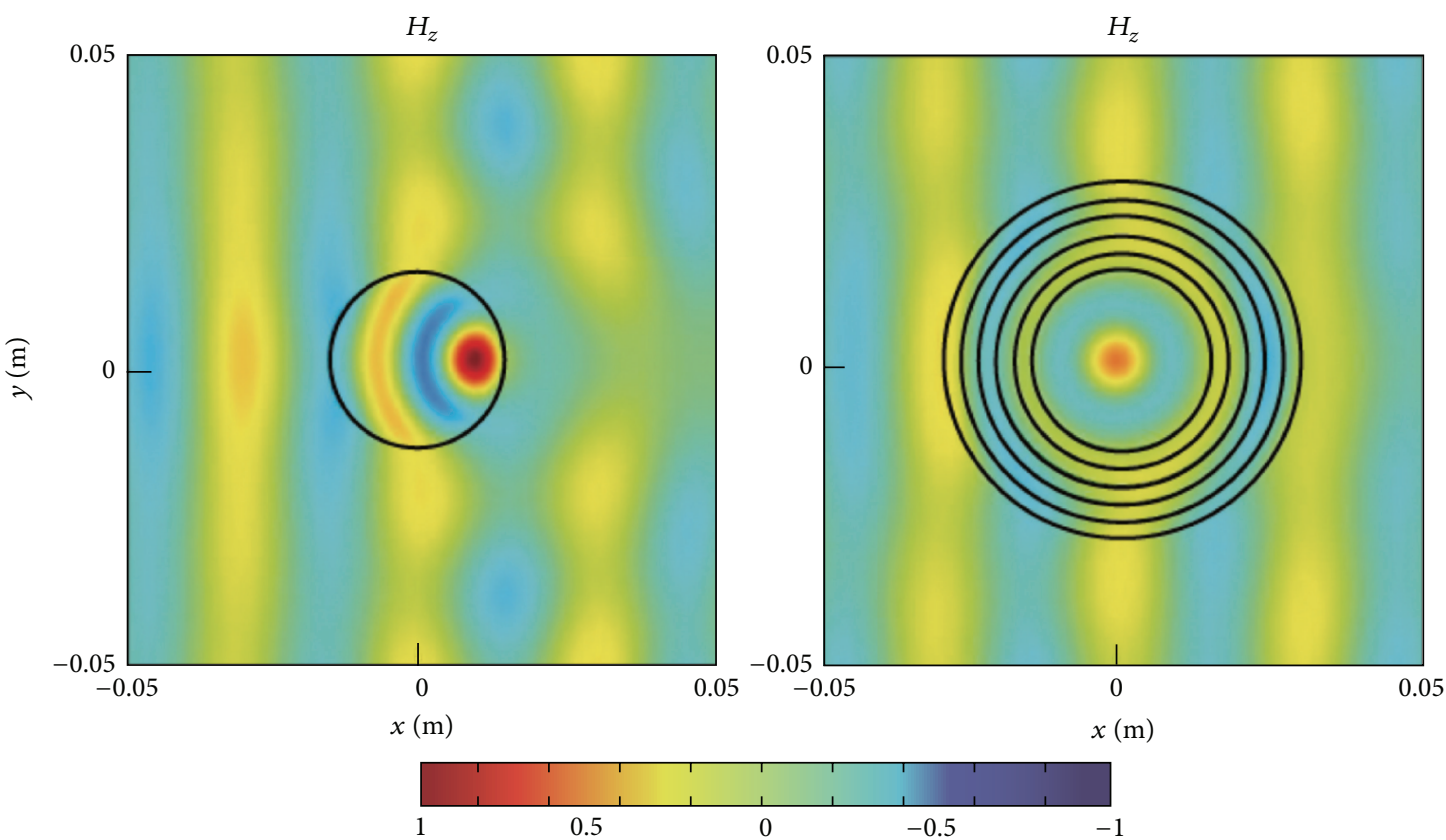

(b)

Figure 9: (a) Configuration of TM mode reconfigurable cloak; independent control over the effective material properties of each unit cell within the cylindrical region is achieved through independent biasing of each column of diodes. (b) Magnetic field distributions surrounding and within the dielectric cylinder with and without the cloak shell at $9.5 \mathrm{GHz}$. The material properties throughout the structure may be tuned to act as a cloak around the inner cylinder. Used by permission [41].

with spatially varying indices of refraction $[34,36,37,72$, $120,132]$. Spatial gradients are the fundamental mechanisms in electromagnetic cloaking. Researchers have demonstrated spatially varying material parameters that are intended to guide electromagnetic waves around a desired region [41, 45, 54]. Wang et al. designed a reconfigurable cloak that could be tuned for multiple operating frequencies. Furthermore, this cloak could be switched from a visible to invisible status [41]. The mechanism for attaining this cloak's spatial gradient index of refraction relaxes fabrication precision constraints because each unit cell can be tuned independently. These features make it ideal for cloaking in RF communication systems [142]. The structure, material parameters, and field distribution of this cloak are depicted in Figure 9. In addition to varying index value, researchers have also demonstrated metamaterials that vary in anisotropy [101]. Such features can be used in creating displays, beamsplitters, isolators, cloaks, and hyperlenses and in controlling luminescence.
4.4. Antenna Propagation Tuning. Tunable metamaterials also provide the potential for increasing existing component functionality and enhancing propagation properties, such as scanning range, radiation pattern, and directivity, while simultaneously reducing costs. In particular, tunable metamaterials have found applications in fabricating reconfigurable directive antennas and beam steering devices, especially for radar and communication systems: radomes, radar absorbent materials, reflectors, electromagnetic interference shielding, and terrestrial and satellite communications. Many of the efforts towards achieving beam steering focus on developing electrically and mechanically tunable high-impedance or artificial magnetic surfaces [24-28, 30, 31, $33,53,64-66,80,95,102-106,109,110,129,143,144]$. Artificial magnetic surfaces typically consist of planar periodic metallic elements backed by a PEC ground plane. Coupled with tuning elements, they are commonly utilized as tunable high impedance ground planes to achieve low-profile 


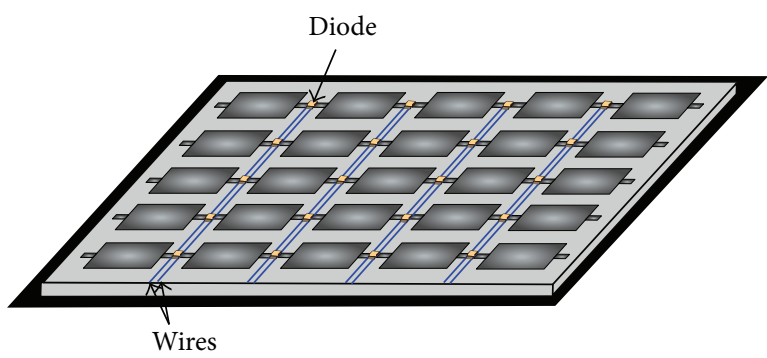

(a)

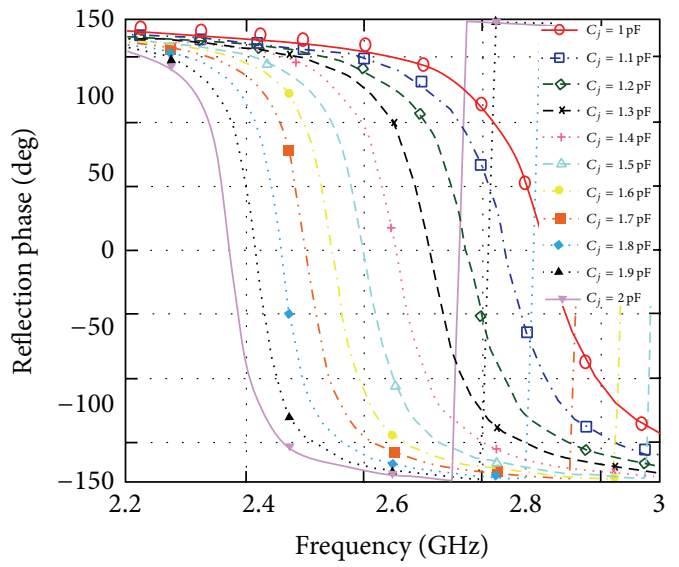

(c)

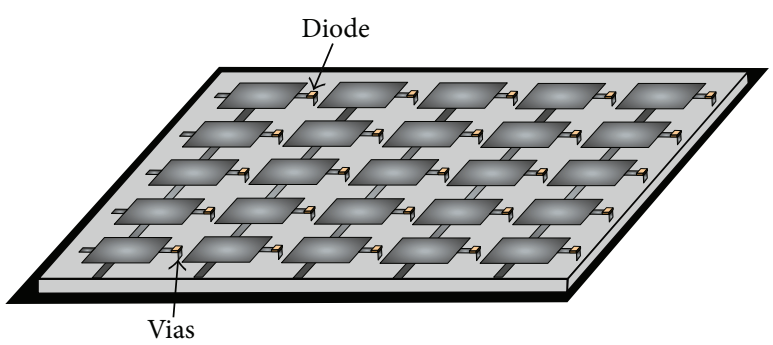

(b)

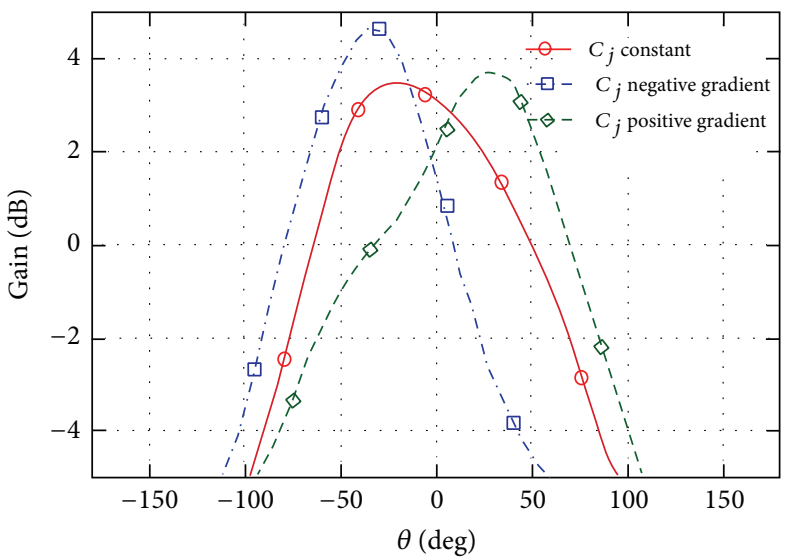

(d)

Figure 10: (a)-(b) Active AMC bow-tie surface showing varactor diode tuning between the bias lines and the vias to the ground plane. (c) Tuning the varactor state changes the frequency response of the AMC. (d) Applying a constant gradient to the AMC capacitances allows beamforming by a bow-tie antenna placed above the surface. Used by permission [64].

antenna configurations. Costa et al. employed this concept to create a steerable bow-tie antenna [64]. Figure 10 displays the artificial magnetic surface, the reflection phase at varied capacitance values, and the resulting beam deflections. Ratajczak et al. demonstrated a directive antenna using a planar electromagnetic band-gap reflector based on the same design principles [28], which is shown in Figure 11. This proposed design offers advantages over traditional directive array antennas, which suffer from complex, expensive, and bulky feeding networks with high losses. This design is easier to fabricate and simple to use in large frequency bands, has low losses due to a waveguide feed structure, and has a tunable radiation pattern. With average tuning ranges of approximately \pm 45 degrees, these tunable impedance surfaces offer low-cost, low-profile, and light-weight alternatives to traditional scanning antennas and reflectors. Similar functionality can be achieved with digitally addressable, anisotropic, and SRR-based metamaterials $[20,76,77,107,121]$. Several papers also document work towards creating partially reflective surfaces that alter the reflection and transmission phases for use in Fabry-Perot cavity systems to obtain directive emissions $[32,40,60,61]$. Tunable metamaterials also offer increased functionality when incorporated into existing devices. Meng et al. demonstrated a reconfigurable magnetic metamaterialloaded waveguide in which the propagation mode could be switched between left-handed and right-handed [108].
This increases the functionality of microwave waveguide systems by allowing designers to broaden the scanning range or control the radiation pattern of leaky wave antennas, which increases the link capacity for multi-input/output communication systems.

\section{Remaining Challenges}

Based on the existing capabilities described in the literature, several broad goals are seen for future developments. Extending tuning concepts that have been demonstrated at an individual or small number of unit cells to tile or fill large regions is necessary for the implementation of many metamaterial or transformation electromagnetics/optics devices, such as lenses. Very few studies have demonstrated spatial tuning of metamaterials for the creation of reconfigurable gradientindex lenses, for example. Expanding the reconfigurable unit cells to large regions of independently tunable elements requires the associated development of tuning and control signal distribution throughout the metamaterial. Finally, extending the tunable range and application flexibility of reconfigurable metamaterial devices will enhance the usefulness and applicability of this design strategy to solving realworld problems.

Another general area for future development is the expansion of available tunable materials. In particular, phasechange materials are under continued development to expand 


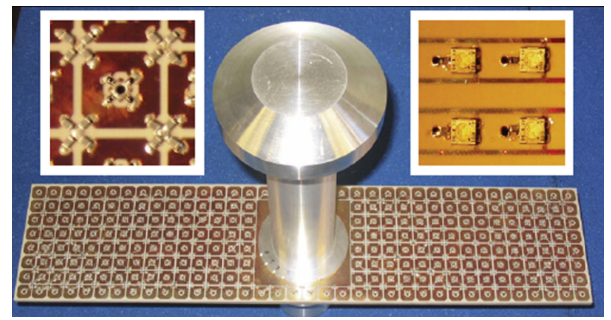

(a)

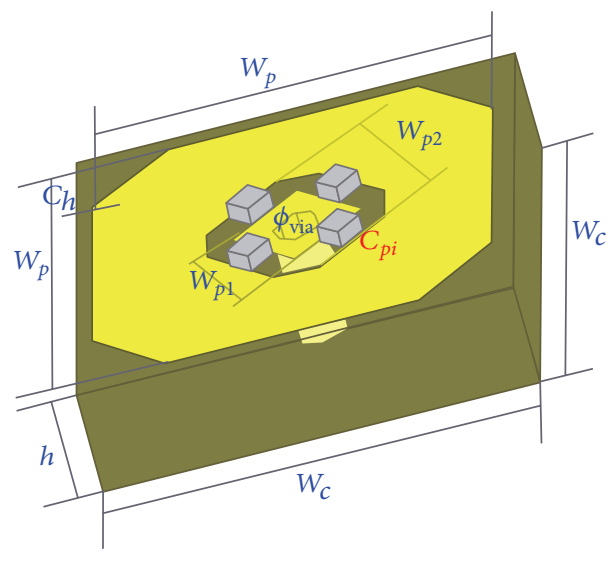

$W_{c}=14.00 \mathrm{~mm}$

$W_{p}=12.80 \mathrm{~mm}$

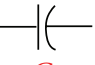

$W_{p 1}=6.00 \mathrm{~mm}$

$W_{p 2}=8.00 \mathrm{~mm}$

$h=3.175 \mathrm{~mm}$

$\varepsilon_{r}=2.2$

$\phi_{\text {via }}=1.5 \mathrm{~mm}$

$C_{h}=3.00 \mathrm{~mm}$

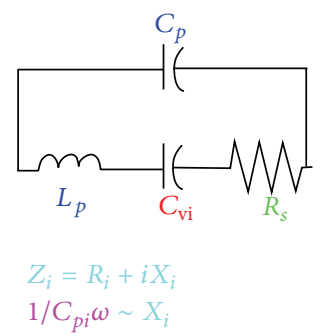

(b)

FIGURE 11: (a) Reconfigurable reflectarray that changes the reflection coefficient from the surface by varying the capacitance between the central via and the ground plane. (b) Reflectarray unit cell geometry and equivalent circuit model. Used by permission [28].

the range of material formulations and to refine the switching and tuning circuitry. While phase-change materials have already been commercialized for high speed memory applications in computer hardware, their suitability for incorporation into practical, high-speed switchable metamaterials is a topic of ongoing research. The development of smart materials that respond to environmental stimuli is also an area of future development. Multiple works were highlighted in the literature looking at conducting polymers that can change properties under stimulus by chemical analytes. Further work is required in both the material science and electromagnetic fields to identify candidate sensing materials and develop metamaterial platforms to bring other smart devices into practice.

Finally, extending the tunable range and application flexibility of reconfigurable metamaterial devices will enhance the usefulness and applicability of this design strategy to solving real-world problems. There is room for advances in material and switch development as well as identifying metamaterial geometries that are sensitive to the switching property in order to increase the device tuning range and functionality.

\section{Conclusion}

In this review, we have extensively documented the state of the metamaterials field as it applies to the generation and usage of tunable or reconfigurable electromagnetic responses. Although metamaterials are themselves in the early stages of development, we have illustrated that the study of tunable metamaterials is a vibrant and active subfield, based on the breadth and depth of the applications and methods that have been reported on in the literature. By classifying the tuning mechanisms and applications into groups based on functionality and capability, rather than operational frequency, our main goal is to provide a comprehensive overview of the state of the art in tunable and reconfigurable metamaterials. New tuning methods and analysis techniques may be applied to existing static metamaterial designs to dramatically increase their capability and effectiveness. Although there is more progress that must be made before many of the techniques discussed here may be practically applied, rapid developments in tunable metamaterials hold great promise for future implementations.

\section{Conflict of Interests}

The authors declare that there is no conflict of interests regarding the publication of this paper.

\section{Acknowledgment}

This work was supported in part by the EMERALD Project, which is funded by the Provincia Autonoma di Trento under the "Bando Unità Ricerca 2011." 


\section{References}

[1] H. Tao, W. J. Padilla, X. Zhang, and R. D. Averitt, "Recent progress in electromagnetic metamaterial devices for terahertz applications," IEEE Journal on Selected Topics in Quantum Electronics, vol. 17, no. 1, pp. 92-101, 2011.

[2] J. B. Pendry, A. J. Holden, D. J. Robbins, and W. J. Stewart, "Magnetism from conductors and enhanced nonlinear phenomena," IEEE Transactions on Microwave Theory and Techniques, vol. 47, no. 11, pp. 2075-2084, 1999.

[3] D. Schurig, J. J. Mock, B. J. Justice et al., "Metamaterial electromagnetic cloak at microwave frequencies," Science, vol. 314, no. 5801, pp. 977-980, 2006.

[4] N. I. Zheludev and Y. S. Kivshar, "From metamaterials to metadevices," Nature Materials, vol. 11, no. 11, pp. 917-924, 2012.

[5] C. L. Holloway, E. F. Kuester, J. A. Gordon, J. O’Hara, J. Booth, and D. R. Smith, "An overview of the theory and applications of metasurfaces: the two-dimensional equivalents of metamaterials," IEEE Antennas and Propagation Magazine, vol. 54, no. 2, pp. 10-35, 2012.

[6] D. J. Kern, D. H. Werner, and M. Lisovich, "Metaferrites: using electromagnetic bandgap structures to synthesize metamaterial ferrites," IEEE Transactions on Antennas and Propagation, vol. 53, no. 4, pp. 1382-1389, 2005.

[7] Z. Bayraktar, M. D. Gregory, X. Wang, and D. H. Werner, "Matched impedance thin planar composite magneto-dielectric metasurfaces," IEEE Transactions on Antennas and Propagation, vol. 60, no. 4, pp. 1910-1920, 2012.

[8] A. Erentok and R. W. Ziolkowski, "Metamaterial-inspired efficient electrically small antennas," IEEE Transactions on Antennas and Propagation, vol. 56, no. 3, pp. 691-707, 2008.

[9] P. Jin and R. W. Ziolkowski, "Broadband, efficient, electrically small metamaterial-inspired antennas facilitated by active nearfield resonant parasitic elements," IEEE Transactions on Antennas and Propagation, vol. 58, no. 2, pp. 318-327, 2010.

[10] C.-Y. Cheng and R. W. Ziolkowski, “Tailoring double-negative metamaterial responses to achieve anomalous propagation effects along microstrip transmission lines," IEEE Transactions on Microwave Theory and Techniques, vol. 51, no. 12, pp. 23062314, 2003.

[11] S. Yun, Z. H. Jiang, Q. Xu, Z. Liu, D. H. Werner, and T. S. Mayer, "Low-loss impedance-matched optical metamaterials with zero-phase delay," ACS Nano, vol. 6, no. 5, pp. 4475-4482, 2012.

[12] M. Barbuto, A. Monti, F. Bilotti, and A. Toscano, "Design of a non-Foster actively loaded SRR and application in metamaterial-inspired components," IEEE Transactions on Antennas and Propagation, vol. 61, no. 3, pp. 1219-1227, 2013.

[13] E. Ozbay, K. Aydin, S. Butun, K. Kolodziejak, and D. Pawlak, "Ferroelectric based tuneable SRR based metamaterial for microwave applications," in Proceedings of the 37th European Microwave Conference (EUMC '07), pp. 497-499, Munich, Germany, October 2007.

[14] A. Vélez, J. Bonache, and F. Martín, "Doubly tuned metamaterial transmission lines based on complementary split-ring resonators," Electromagnetics, vol. 28, no. 7, pp. 523-530, 2008.

[15] A. Velez, J. Bonache, and F. Martin, "Varactor-loaded complementary split ring resonators (VLCSRR) and their application to tunable metamaterial transmission lines," IEEE Microwave and Wireless Components Letters, vol. 18, no. 1, pp. 28-30, 2008.
[16] H. Lim, W.-S. Jeong, S.-H. Lim, D.-H. Shin, and N.-H. Myung, "A tunable notch resonator based on varactor-loaded complementary split-ring resonators," in Proceedings of the IEEE International Workshop on Antenna Technology (iWAT '08), pp. 426-429, Chiba, Japan, 2008.

[17] H.-T. Chen, W. J. Padilla, J. M. O. Zide, A. C. Gossard, A. J. Taylor, and R. D. Averitt, "Active terahertz metamaterial devices," Nature, vol. 444, no. 7119, pp. 597-600, 2006.

[18] J. Han and A. Lakhtakia, "Semiconductor split-ring resonators for thermally tunable terahertz metamaterials," Journal of Modern Optics, vol. 56, no. 4, pp. 554-557, 2009.

[19] J. Han, A. Lakhtakia, and C.-W. Qiu, "Terahertz metamaterials with semiconductor split-ring resonators for magnetostatic tunability," Optics Express, vol. 16, no. 19, pp. 14390-14396, 2008.

[20] T. H. Hand and S. A. Cummer, "Reconfigurable reflectarray using addressable metamaterials," IEEE Antennas and Wireless Propagation Letters, vol. 9, pp. 70-74, 2010.

[21] D. Shrekenhamer, S. Rout, A. C. Strikwerda et al., "High speed terahertz modulation from metamaterials with embedded high electron mobility transistors," Optics Express, vol. 19, no. 10, pp. 9968-9975, 2011.

[22] E. Ekmekci, K. Topalli, T. Akin, and G. Turhan-Sayan, "A tunable multi-band metamaterial design using micro-split SRR structures," Optics Express, vol. 17, no. 18, pp. 16046-16058, 2009.

[23] I. Gil, J. Bonache, J. García-García, and F. Martín, "Tunable metamaterial transmission lines based on varactor-loaded splitring resonators," IEEE Transactions on Microwave Theory and Techniques, vol. 54, no. 6, pp. 2665-2674, 2006.

[24] D. Sievenpiper and J. Schaffner, "Beam steering microwave reflector based on electrically tunable impedance surface," Electronics Letters, vol. 38, no. 21, pp. 1237-1238, 2002.

[25] D. Sievenpiper, J. Schaffner, B. Loo et al., "Electronic beam steering using a varactor-tuned impedance surface," in Proceedings of the IEEE Antennas and Propagation Society International Symposium, pp. 174-177, Boston, Mass, USA, July 2001.

[26] D. F. Sievenpiper, "Forward and backward leaky wave radiation with large effective aperture from an electronically tunable textured surface," IEEE Transactions on Antennas and Propagation, vol. 53, no. 1, pp. 236-247, 2005.

[27] D. F. Sievenpiper, J. H. Schaffner, H. Jae Song, R. Y. Loo, and G. Tangonan, "Two-dimensional beam steering using an electrically tunable impedance surface," IEEE Transactions on Antennas and Propagation, vol. 51, no. 10, pp. 2713-2722, 2003.

[28] P. Ratajczak, P. Brachat, and J.-M. Baracco, "Active reflectarray based on high impedance surface," in Proceedings of the IEEE Antennas and Propagation Society International Symposium, pp. 5327-5330, Honolulu, Hawaii, USA, June 2007.

[29] C. Mias and J. H. Yap, "A varactor-tunable high impedance surface with resistive-lumped-element biasing grid," IEEE Transactions on Antennas and Propagation, vol. 55, no. 7, pp. 1955-1962, 2007.

[30] J. A. Higgins, H. Xin, A. Sailer, and M. Rosker, "Ka-band waveguide phase shifter using tunable electromagnetic crystal sidewalls," IEEE Transactions on Microwave Theory and Techniques, vol. 51, no. 4, pp. 1281-1288, 2003.

[31] F. Costa, A. Monorchio, and G. P. Vastante, "Tunable highimpedance surface with a reduced number of varactors," IEEE Antennas and Wireless Propagation Letters, vol. 10, pp. 11-13, 2011.

[32] F. Costa and A. Monorchio, "Design of subwavelength tunable and steer-able Fabry-Perot/leaky wave antennas," Progress in Electromagnetics Research, vol. 111, pp. 467-481, 2011. 
[33] F. Costa, A. Monorchio, S. Talarico, and F. M. Valeri, "An active high-impedance surface for low-profile tunable and steerable antennas," IEEE Antennas and Wireless Propagation Letters, vol. 7, pp. 676-680, 2008.

[34] D. H. Werner, D.-H. Kwon, I.-C. Khoo, A. V. Kildishev, and V. M. Shalaev, "Liquid crystal clad near-infrared metamaterials with tunable negative-zero-positive refractive indices," Optics Express, vol. 15, no. 6, pp. 3342-3347, 2007.

[35] D. F. Gardner, J. S. Evans, and I. I. Smalyukh, "Towards reconfigurable optical metamaterials: colloidal nanoparticle self-assembly and self-alignment in liquid crystals," Molecular Crystals and Liquid Crystals, vol. 545, pp. 3-21, 2011.

[36] A. B. Golovin and O. D. Lavrentovich, "Electrically reconfigurable optical metamaterial based on colloidal dispersion of metal nanorods in dielectric fluid," Applied Physics Letters, vol. 95, no. 25, Article ID 254104, 2009.

[37] A. B. Golovin, J. Xiang, Y. A. Nastishin, and O. D. Lavrentovich, "Electrically reconfigurable optical metamaterials based on orientationally ordered dispersions of metal nano-rods in dielectric fluids," in Liquid Crystals XIV, vol. 7775 of Proceedings of SPIE, pp. 1-14, San Diego, Calif, USA, August 2010.

[38] J.-Y. Ou, E. Plum, J. Zhang, and N. I. Zheludev, "An electromechanically reconfigurable plasmonic metamaterial operating in the near-infrared," Nature Nanotechnology, vol. 8, no. 4, pp. 252255,2013

[39] F. Bayatpur and K. Sarabandi, "A tunable metamaterial frequency-selective surface with variable modes of operation," IEEE Transactions on Microwave Theory and Techniques, vol. 57, no. 6, pp. 1433-1438, 2009.

[40] S. N. Burokur, J.-P. Daniel, P. Ratajczak, and A. De Lustrac, "Tunable bilayered metasurface for frequency reconfigurable directive emissions," Applied Physics Letters, vol. 97, no. 6, Article ID 064101, 3 pages, 2010.

[41] D. Wang, H. Chen, L. Ran, J. Huangfu, J. A. Kong, and B.-I. $\mathrm{Wu}$, "Reconfigurable cloak for multiple operating frequencies," Applied Physics Letters, vol. 93, no. 4, Article ID 043515, 3 pages, 2008.

[42] J. A. Bossard, X. Liang, L. Li et al., "Tunable frequency selective surfaces and negative-zero-positive index metamaterials based on liquid crystals," IEEE Transactions on Antennas and Propagation, vol. 56, no. 5, pp. 1308-1320, 2008.

[43] D. H. Werner, T. S. Mayer, C. Rivero-Baleine et al., "Adaptive phase change metamaterials for infrared aperture control," in Unconventional Imaging, Wavefront Sensing, and Adaptive Coded Aperture Imaging and Non-Imaging Sensor Systems, vol. 8165 of Proceedings of SPIE, pp. 1-9, August 2011.

[44] A. Yu, F. Yang, and A. Elsherbeni, "A dual band circularly polarized ring antenna based on composite right and left handed metamaterials," Progress in Electromagnetics Research, vol. 78, pp. 73-81, 2008.

[45] S. Hrabar, I. Krois, and A. Kiricenko, "Towards active dispersionless ENZ metamaterial for cloaking applications," Metamaterials, vol. 4, no. 2-3, pp. 89-97, 2010.

[46] H. Mirzaei and G. V. Eleftheriades, "A compact frequencyreconfigurable metamaterial-inspired antenna," IEEE Antennas and Wireless Propagation Letters, vol. 10, pp. 1154-1157, 2011.

[47] J. Choi and C. Seo, "Broadband and low phase noise VCO using tunable metamaterial transmission line based on varactorloaded split-ring resonator," in Proceedings of the Korea-Japan MicroWave Conference (KJMW '07), pp. 145-148, Okinawa, Japan, November 2007.
[48] A. Vélez, J. Bonache, and F. Martín, "Effects of varying the series capacitance in CSRR-loaded metamaterial transmission lines," Microwave and Optical Technology Letters, vol. 49, no. 9, pp. 2245-2248, 2007.

[49] H. Li, Y. Zhang, and L. He, "Tunable filters based on the varactor-loaded spilt-ring resonant structure coupled to the micropstrip line," in Proceedings of the International Conference on Microwave and Millimeter Wave Technology (ICMMT '08), pp. 1580-1582, Nanjing, China, April 2008.

[50] I. Gil, J. Bonache, J. García-García, F. Martín, and R. Marqués, "Tunable split rings resonators for reconfigurable metamaterial transmission lines," in Proceedings of the European Microwave Conference, vol. 2, pp. 905-908, October 2005.

[51] I. Gil, J. García-García, J. Bonache, F. Martín, M. Sorolla, and R. Marqués, "Varactor-loaded split ring resonators for tunable notch filters at microwave frequencies," Electronics Letters, vol. 40, no. 21, pp. 1347-1348, 2004.

[52] C. Mias, "Varactor-tunable frequency selective surface with resistive-lumped-element biasing grids," IEEE Microwave and Wireless Components Letters, vol. 15, no. 9, pp. 570-572, 2005.

[53] R. J. Langley, H.-J. Lee, and K. L. Ford, "Independent multiband tuning using an active AMC," in Proceedings of the 1st IEEEAPS Topical Conference on Antennas and Propagation in Wireless Communications (APWC '11), pp. 576-579, Torino, Italy, September 2011.

[54] D. Wang, L. Ran, H. Chen, M. Mu, J. A. Kong, and B.-I. $\mathrm{Wu}$, "Active left-handed material collaborated with microwave varactors," Applied Physics Letters, vol. 91, no. 16, Article ID 164101, 3 pages, 2007.

[55] D. Huang, E. Poutrina, and D. R. Smith, "Analysis of the power dependent tuning of a varactor-loaded metamaterial at microwave frequencies," Applied Physics Letters, vol. 96, no. 10, Article ID 104104, 3 pages, 2010.

[56] I. V. Shadrivov, S. K. Morrison, and Y. S. Kivshar, "Tunable splitring resonators for nonlinear negative-index metamaterials," Optics Express, vol. 14, no. 20, pp. 9344-9349, 2006.

[57] O. Reynet and O. Acher, "Voltage controlled metamaterial," Applied Physics Letters, vol. 84, no. 7, pp. 1198-1200, 2004.

[58] H.-J. Lee, K. L. Ford, and R. J. Langley, "Independently tunable low-profile dual-band high-impedance surface antenna system for applications in UHF band," IEEE Transactions on Antennas and Propagation, vol. 60, no. 9, pp. 4092-4101, 2012.

[59] J. Liang and H. Y. D. Yang, "Microstrip patch antennas on tunable electromagnetic band-gap substrates," IEEE Transactions on Antennas and Propagation, vol. 57, no. 6, pp. 1612-1617, 2009.

[60] A. R. Weily, T. S. Bird, and Y. J. Guo, "A reconfigurable highgain partially reflecting surface antenna," IEEE Transactions on Antennas and Propagation, vol. 56, no. 11, pp. 3382-3390, 2008.

[61] A. Ourir, S. N. Burokur, and A. De Lustrac, "Electronic beam steering of an active metamaterial-based directive subwavelength cavity," in Proceedings of the 2nd European Conference on Antennas and Propagation (EuCAP '07), p. 257, Edinburgh, UK, November 2007.

[62] H. Mirzaei and G. V. Eleftheriades, "A wideband metamaterialinspired compact antenna using embedded non-Foster matching," in Proceedings of the IEEE International Symposium on Antennas and Propagation, pp. 1950-1953, Spokane, Wash, USA, July 2011.

[63] N. Wiwatcharagoses and P. Chahal, "A novel reconfigurable metamaterial unit cell based composite right/left handed microstrip design," in Proceedings of the IEEE International 
Symposium on Antennas and Propagation, pp. 2954-2957, Spokane, Wash, USA, July 2011.

[64] F. Costa, S. Talarico, A. Monorchio, and M. F. Valeri, "An active AMC ground plane for tunable low-profile antennas," in Proceedings of the IEEE International Symposium on Antennas and Propagation, pp. 1-4, San Diego, Calif, USA, July 2008.

[65] K. L. Ford and J. M. Rigelsford, "Dipole radiation steering using an active artificial magnetic conductor," in Proceedings of the IEEE International Symposium on Antennas and Propagation, pp. 1-4, July 2008.

[66] M. G. Bray, Z. Bayraktar, and D. H. Werner, "GA optimized ultra-thin tunable EBG AMC surfaces," in Proceedings of the IEEE Antennas and Propagation Society International Symposium, pp. 410-413, Albuquerque, NM, USA, July 2006.

[67] C. Huang, Z. Zhao, and X. Luo, "Tuning enhanced transmission frequency through a subwavelength aperture with active split ring resonator," in Proceedings of the 5th International Symposium on Advanced Optical Manufacturing and Testing Technologies: Smart Structures and Materials in Manufacturing and Testing, vol. 7659 of Proceedings of SPIE, pp. 1-5, April 2010.

[68] I. Martinez, A. H. Panaretos, D. H. Werner, G. Oliveri, and A. Massa, "Ultra-thin reconfigurable electromagnetic metasurface absorbers," in Proceedings of the 7th European Conference on Antennas and Propagation (EuCAP '13), pp. 1843-1847, 2013.

[69] A. R. Katko, A. M. Hawkes, J. P. Barrett, and S. A. Cummer, "RF limiter metamaterial using p-i-n diodes," IEEE Antennas and Wireless Propagation Letters, vol. 10, pp. 1571-1574, 2011.

[70] Y. Kotsuka and C. Kawamura, "Novel computer controllable metamaterial beyond conventional configurations and its microwave absorber application," in Proceedings of the IEEE MTT-S International Microwave Symposium (IMS '07), pp. 1627-1630, Honolulu, Hawaii, USA, June 2007.

[71] P. Ratajczak, P. Brachat, and J. M. Fargeas, "An adaptive beam steering antenna for mobile communications," in Proceedings of the IEEE Antennas and Propagation Society International Symposium, pp. 418-421, Albuquerque, NM, USA, July 2006.

[72] T. H. Hand and S. A. Cummer, "Controllable magnetic metamaterial using digitally addressable split-ring resonators," IEEE Antennas and Wireless Propagation Letters, vol. 8, pp. 262-265, 2009.

[73] J. Choi and S. Lim, "Frequency reconfigurable metamaterial resonant antenna," in Proceedings of the Asia Pacific Microwave Conference (APMC '09), pp. 798-801, Singapore, December 2009.

[74] K. B. Alici, F. Bilotti, L. Vegni, and E. Ozbay, "Optimization and tunability of deep subwavelength resonators for metamaterial applications: complete enhanced transmission through a subwavelength aperture," Optics Express, vol. 17, no. 8, pp. 5933$5943,2009$.

[75] T. Liang, L. Li, J. A. Bossard, D. H. Werner, and T. S. Mayer, "Reconfigurable ultra-thin EBG absorbers using conducting polymers," in Proceedings of the IEEE Antennas and Propagation Society International Symposium, vol. 2, pp. 204-207, July 2005.

[76] I. O. Mirza, S. Shi, and D. W. Prather, "Phase modulation using dual split ring resonators," Optics Express, vol. 17, no. 7, pp. 50895097, 2009.

[77] I. O. Mirza, J. N. Sabas, S. Shi, and D. W. Prather, "Experimental demonstration of metamaterial-based phase modulation," Progress in Electromagnetics Research, vol. 93, pp. 1-12, 2009.

[78] V. Sanchez and E. Paller, "A tunable artificial magnetic conductor using switched capacitance in a concentric overlapping geometry," in Proceedings of the IEEE International Antennas and Propagation Symposium, vol. 2, pp. 439-442, Columbus, Ohio, USA, June 2003.

[79] D. Bouyge, A. Crunteanu, A. Pothier et al., "Reconfigurable 4 pole bandstop filter based on RF-MEMS-loaded split ring resonators," in Proceedings of the IEEE MTT-S International Microwave Symposium (MTT '10), pp. 588-591, May 2010.

[80] T. Hand and S. Cummer, "Characterization of tunable metamaterial elements using MEMS switches," IEEE Antennas and Wireless Propagation Letters, vol. 6, pp. 401-404, 2007.

[81] D. Bouyge, A. Crunteanu, M. Duran-Sindreu et al., "Reconfigurable split rings based on MEMS switches and their application to tunable filters," Journal of Optics, vol. 14, no. 11, Article ID 114001, 9 pages, 2012.

[82] J. G. Linvill, “Transistor negative-impedance converters," Proceedings of the IRE, vol. 41, no. 6, pp. 725-729, 1953.

[83] D. J. Kern, D. H. Werner, and M. J. Wilhelm, "Active negative impedance loaded EBG structures for the realization of ultrawideband artificial magnetic conductors," in Proceedings of the IEEE International Antennas and Propagation Symposium, pp. 427-430, Columbus, Ohio, USA, June 2003.

[84] S. E. Sussman-Fort and R. M. Rudish, "Non-foster impedance matching of electrically-small antennas," IEEE Transactions on Antennas and Propagation, vol. 57, no. 8, pp. 2230-2241, 2009.

[85] B.-I. Popa and S. A. Cummer, "An architecture for active metamaterial particles and experimental validation at RF," Microwave and Optical Technology Letters, vol. 49, no. 10, pp. 2574-2577, 2007.

[86] S. Wuestner, A. Pusch, K. L. Tsakmakidis, J. M. Hamm, and O. Hess, "Overcoming losses with gain in a negative refractive index metamaterial," Physical Review Letters, vol. 105, no. 12, Article ID 127401, 4 pages, 2010.

[87] D. J. Gregoire, C. R. White, and J. S. Colburn, "Wideband artificial magnetic conductors loaded with non-foster negative inductors," IEEE Antennas and Wireless Propagation Letters, vol. 10, pp. 1586-1589, 2011.

[88] K. Z. Rajab, Y. Hao, D. Bao, C. G. Parini, J. Vazquez, and M. Philippakis, "Stability of active magnetoinductive metamaterials," Journal of Applied Physics, vol. 108, no. 5, Article ID 054904, 6 pages, 2010.

[89] S. Hrabar, I. Krois, I. Bonic, and A. Kiricenko, "Negative capacitor paves the way to ultra-broadband metamaterials," Applied Physics Letters, vol. 99, no. 25, Article ID 254103, 4 pages, 2011.

[90] Q. Liu, Y. Cui, D. Gardner, X. Li, S. He, and I. I. Smalyukh, "Self-alignment of plasmonic gold nanorods in reconfigurable anisotropic fluids for tunable bulk metamaterial applications," Nano Letters, vol. 10, no. 4, pp. 1347-1353, 2010.

[91] H. Tao, A. C. Strikwerda, K. Fan, W. J. Padilla, X. Zhang, and R. D. Averitt, "MEMS based structurally tunable metamaterials at terahertz frequencies," Journal of Infrared, Millimeter, and Terahertz Waves, vol. 32, no. 5, pp. 580-595, 2011.

[92] H. Tao, A. C. Strikwerda, K. Fan, W. J. Padilla, X. Zhang, and R. D. Averitt, "Reconfigurable terahertz metamaterials," Physical Review Letters, vol. 103, no. 14, Article ID 147401, 4 pages, 2009.

[93] I. Gil, F. Martin, X. Rottenberg, and W. De Raedt, "Tunable stop-band filter at Q-band based on RF-MEMS metamaterials," Electronics Letters, vol. 43, no. 21, pp. 1153-1154, 2007.

[94] K. Fuchi, A. R. Diaz, E. J. Rothwell, R. O. Ouedraogo, and J. Tang, "An origami tunable metamaterial," Journal of Applied Physics, vol. 111, no. 8, Article ID 084905, 7 pages, 2012. 
[95] J. Sanz-Fernández, G. Goussetis, and R. Cheung, “Tunable 2D electromagnetic band-gap (EBG) structures based on microelectro-mechanical systems (MEMS) for THz frequencies," in Proceedings of the IEEE International Symposium on Antennas and Propagation, pp. 1-4, Toronto, Canada, July 2010.

[96] Y. H. Fu, A. Q. Liu, W. M. Zhu et al., "A micromachined reconfigurable metamaterial via reconfiguration of asymmetric split-ring resonators," Advanced Functional Materials, vol. 21, no. 18, pp. 3589-3594, 2011.

[97] I. M. Pryce, K. Aydin, Y. A. Kelaita, R. M. Briggs, and H. A. Atwater, "Highly strained compliant optical metamaterials with large frequency tunability," Nano Letters, vol. 10, no. 10, pp. 4222-4227, 2010.

[98] W. Zhang, A. Q. Liu, W. M. Zhu et al., "Micromachined switchable metamaterial with dual resonance," Applied Physics Letters, vol. 101, no. 15, Article ID 151902, 4 pages.

[99] J. Y. Ou, E. Plum, L. Jiang, and N. I. Zheludev, "Reconfigurable photonic metamaterials," Nano Letters, vol. 11, no. 5, pp. 21422144, 2011.

[100] D. Dubuc, K. Grenier, H. Fujita, and H. Toshiyoshi, "Microfabricated tunable artificial dielectric for reconfigurable microwave circuits," in Proceedings of the 39th European Microwave Conference (EuMC '09), pp. 520-523, October 2009.

[101] W. M. Zhu, A. Q. Liu, T. Bourouina et al., "Microelectromechanical Maltese-cross metamaterial with tunable terahertz anisotropy," Nature Communications, vol. 3, p. 1274, 2012.

[102] D. Sievenpiper, J. Schaffner, J. J. Lee, and S. Livingston, “A steerable leaky-wave antenna using a tunable impedance ground plane," IEEE Antennas and Wireless Propagation Letters, vol. 1, pp. 179-182, 2002.

[103] D. Chicherin, S. Dudorov, D. Lioubtchenko, V. Ovchinnikov, S. Tretyakov, and A. V. Räisänen, "Mems-based high-impedance surfaces for millimeter and submillimeter wave applications," Microwave and Optical Technology Letters, vol. 48, no. 12, pp. 2570-2573, 2006.

[104] D. Chicherin, S. Dudorov, D. Lioubtchenko, V. Ovchinnikov, and A. V. Räisänen, "Millimetre wave phase shifters based on a metal waveguide with a MEMS-based high-impedance surface," in Proceedings of the 36th European Microwave Conference (EuMC '06), pp. 372-375, Manchester, UK, September 2006.

[105] M. Sterner, D. Chicherin, A. V. Riisenen, G. Stemme, and J. Oberhammer, "RF MEMS high-impedance tuneable metamaterials for millimeter-wave beam steering," in Proceedings of the 22nd IEEE International Conference on Micro Electro Mechanical Systems (MEMS '09), pp. 896-899, Sorrento, Italy, January 2009.

[106] D. Chicherini, M. Sterner, J. Oberhammer, S. Dudorovi, J. Åberg, and A. V. Raisanen, "Analog type millimeter wave phase shifters based on MEMS tunable high-impedance surface in rectangular metal waveguide," in Proceedings of the IEEE MTT-S International Microwave Symposium (MTT '10), pp. 61-64, May 2010.

[107] P. E. Sieber and D. H. Werner, "A reconfigurable nearinfrared circularly polarizing reflector based on phase changing anisotropic metamaterials," in Proceedings of the IEEE Antennas and Propagation Society International Symposium, pp. 1-2, Chicago, Ill, USA, 2012.

[108] F.-Y. Meng, K. Zhang, Q. Wu, and L. Jong-Chul, "Reconfigurable composite right/left-handed magnetic-metamaterial waveguide at sub-wavelength scale," Journal of Applied Physics, vol. 109, no. 7, Article ID 07A309, 3 pages, 2011.
[109] D. Sievenpiper, J. Schaffner, R. Loo, G. Tangonan, S. Ontiveros, and R. Harold, "A tunable impedance surface performing as a reconfigurable beam steering reflector," IEEE Transactions on Antennas and Propagation, vol. 50, no. 3, pp. 384-390, 2002.

[110] D. Chicherin, M. Sterner, D. Lioubtchenko, J. Oberhammer, and A. V. Räisänen, "Analog-type millimeter-wave phase shifters based on MEMS tunable high-impedance surface and dielectric rod waveguide," International Journal of Microwave and Wireless Technologies, vol. 3, no. 5, pp. 533-538, 2011.

[111] T. S. Kasirga, Y. N. Ertas, and M. Bayindir, "Microfluidics for reconfigurable electromagnetic metamaterials," Applied Physics Letters, vol. 95, no. 21, Article ID 214102, 3 pages, 2009.

[112] D. J. Kern, M. J. Wilhelm, D. H. Werner, and P. L. Werner, "A novel design technique for ultra-thin tunable EBG AMC surfaces," in Proceedings of the IEEE Antennas and Propagation Society Symposium, vol. 2, pp. 1167-1170, June 2004.

[113] Z. Sheng and V. V. Varadan, "Effect of substrate dielectric properties and tunable metamaterials," in Proceedings of the IEEE Antennas and Propagation Society International Symposium, pp. 4497-4500, 2006.

[114] Z. Sheng and V. V. Varadan, "Tuning the effective properties of metamaterials by changing the substrate properties," Journal of Applied Physics, vol. 101, no. 1, Article ID 014909, 7 pages, 2007.

[115] G. Houzet, X. Mélique, D. Lippens, L. Burgnies, G. Velu, and J.-C. Carru, "Microstrip transmission line loaded by splitring resonators tuned by ferroelectric thin film," Progress In Electromagnetics Research C, vol. 12, pp. 225-236, 2010.

[116] M. Gil, C. Damm, A. Giere et al., "Electrically tunable splitring resonators at microwave frequencies based on bariumstrontium-titanate thick films," Electronics Letters, vol. 45, no. 8, pp. 417-418, 2009.

[117] Q. Zhao, L. Kang, B. Du et al., "Electrically tunable negative permeability metamaterials based on nematic liquid crystals," Applied Physics Letters, vol. 90, no. 1, Article ID 011112, 3 pages, 2007.

[118] F. Zhang, Q. Zhao, L. Kang et al., "Magnetic control of negative permeability metamaterials based on liquid crystals," in Proceedings of the 38th European Microwave Conference (EuMC '08), pp. 801-804, Amsterdam, The Netherlands, October 2008.

[119] D.-H. Kwon, X. Wang, Z. Bayraktar, B. Weiner, and D. H. Werner, "Near-infrared metamaterial films with reconfigurable transmissive/ reflective properties," Optics Letters, vol. 33, no. 6, pp. 545-547, 2008.

[120] X. Wang, D.-H. Kwon, D. H. Werner, I.-C. Khoo, A. V. Kildishev, and V. M. Shalaev, "Tunable optical negative-index metamaterials employing anisotropic liquid crystals," Applied Physics Letters, vol. 91, no. 14, Article ID 143122, 2007.

[121] P. E. Sieber and D. H. Werner, "Reconfigurable broadband infrared circularly polarizing reflectors based on phase changing birefringent metasurfaces," Optics Express, vol. 21, no. 1, pp. 1087-1100, 2013.

[122] L. Kang, Q. Zhao, H. Zhao, and J. Zhou, "Magnetically tunable negative permeability metamaterial composed by split ring resonators and ferrite rods," Optics Express, vol. 16, no. 12, pp. 8825-8834, 2008.

[123] S. Zhu, D. G. Holtby, K. L. Ford, A. Tennant, and R. J. Langley, "Compact low frequency varactor loaded tunable SRR antenna," IEEE Transactions on Antennas and Propagation, vol. 61, no. 4, pp. 2301-2304, 2013. 
[124] K. A. Boulais, D. W. Rule, S. Simmons et al., "Tunable split-ring resonator for metamaterials using photocapacitance of semiinsulating GaAs," Applied Physics Letters, vol. 93, no. 4, Article ID 043518, 3 pages, 2008.

[125] H.-T. Chen, J. F. O’Hara, A. K. Azad et al., "Experimental demonstration of frequency-agile terahertz metamaterials," Nature Photonics, vol. 2, no. 5, pp. 295-298, 2008.

[126] S. Xiao, U. K. Chettiar, A. V. Kildishev, V. Drachev, I. C. Khoo, and V. M. Shalaev, "Tunable magnetic response of metamaterials," Applied Physics Letters, vol. 95, no. 3, Article ID 033115, 3 pages, 2009.

[127] D. Roy Chowdhury, R. Singh, J. F. O’Hara, H.-T. Chen, A. J. Taylor, and A. K. Azad, "Dynamically reconfigurable terahertz metamaterial through photo-doped semiconductor," Applied Physics Letters, vol. 99, no. 23, Article ID 231101, 3 pages, 2011.

[128] W. J. Padilla, A. J. Taylor, C. Highstrete, M. Lee, and R. D. Averitt, "Dynamical electric and magnetic metamaterial response at terahertz frequencies," Physical Review Letters, vol. 96, no. 10, Article ID 107401, 4 pages, 2006.

[129] Y. Huang, L.-S. Wu, M. Tang, and J. Mao, "Design of a beam reconfigurable $\mathrm{THz}$ antenna with graphene-based switchable high-impedance surface," IEEE Transactions on Nanotechnology, vol. 11, no. 4, pp. 836-842, 2012.

[130] D. Bouyge, A. Crunteanu, J.-C. Orlianges et al., "Reconfigurable bandpass filter based on split ring resonators and vanadium dioxide (VO2) microwave switches," in Proceedings of the Asia Pacific Microwave Conference (APMC '09), pp. 2332-2335, Singapore, December 2009.

[131] D. Bouyge, A. Crunteanu, O. Massagué et al., "Applications of vanadium dioxide (VO2)-loaded electrically small resonators in the design of tunable filters," in Proceedings of the 40th European Microwave Conference (EuMC '10), pp. 822-825, September 2010.

[132] M. D. Goldflam, T. Driscoll, B. Chapler et al., "Reconfigurable gradient index using VO2 memory metamaterials," Applied Physics Letters, vol. 99, no. 4, Article ID 044103, 3 pages, 2011.

[133] E. Pistono, P. Ferrari, L. Duvillaret, J.-M. Duchamp, and R. G. Harrison, "Hybrid narrow-band tunable bandpass filter based on varactor loaded electromagnetic-bandgap coplanar waveguides," IEEE Transactions on Microwave Theory and Techniques, vol. 53, no. 8, pp. 2506-2514, 2005.

[134] M. G. Bray and D. H. Werner, "A broadband open-sleeve dipole antenna mounted above a tunable EBG AMC ground plane," in Proceedings of the IEEE Antennas and Propagation Society Symposium, pp. 1147-1150, Monterey, Calif, USA, June 2004.

[135] R. Langley, L. Liu, H.-J. Lee, and L. Ford, “Tunable antennas and AMC structures," in Proceedings of the IEEE Antennas and Propagation Society International Symposium, pp. 1-4, Toronto, Canada, 2010.

[136] F. Zhang, L. Kang, Q. Zhao, J. Zhou, X. Zhao, and D. Lippens, "Magnetically tunable left handed metamaterials by liquid crystal orientation," Optics Express, vol. 17, no. 6, pp. 4360-4366, 2009.

[137] C. Jeppesen, S. Xiao, N. A. Mortensen, and A. Kristensen, "Capacitance tuning of nanoscale split-ring resonators," in Metamaterials V, vol. 7711 of Proceedings of SPIE, pp. 1-6, April 2010.

[138] D. A. Powell, A. Alù, B. Edwards, A. Vakil, Y. S. Kivshar, and N. Engheta, "Nonlinear control of tunneling through an epsilonnear-zero channel," Physical Review B, vol. 79, no. 24, Article ID 245135, 2009.
[139] D. J. Kern, J. A. Bossard, and D. H. Werner, "Design of reconfigurable electromagnetic bandgap surfaces as artificial magnetic conducting ground planes and absorbers," in Proceedings of the IEEE Antennas and Propagation Society International Symposium, pp. 197-200, Albuquerque, NM, USA, July 2006.

[140] D.-H. Kwon and D. H. Werner, "Transformation electromagnetics: an overview of the theory and applications," IEEE Antennas and Propagation Magazine, vol. 52, no. 1, pp. 24-46, 2010.

[141] D. H. Werner and D.-H. Kwon, Eds., Transformation Electromagnetics and Metamaterials, Springer, London, UK, 2013.

[142] D.-H. Kwon and D. H. Werner, "Restoration of antenna parameters in scattering environments using electromagnetic cloaking," Applied Physics Letters, vol. 92, no. 11, Article ID 113507, 3 pages, 2008.

[143] M. G. Bray and D. H. Werner, "A novel design approach for an independently tunable dual-band EBG AMC surface," in Proceedings of the IEEE Antennas and Propagation Society Symposium, pp. 289-292, Monterey, Calif, USA, June 2004.

[144] J. M. Rigelsford, F. Collado, and K. L. Ford, "Radiation steering of a low profile street furniture antenna using an active AMC," in Proceedings of the 6th Loughborough Antennas and Propagation Conference (LAPC '10), pp. 529-531, November 2010. 

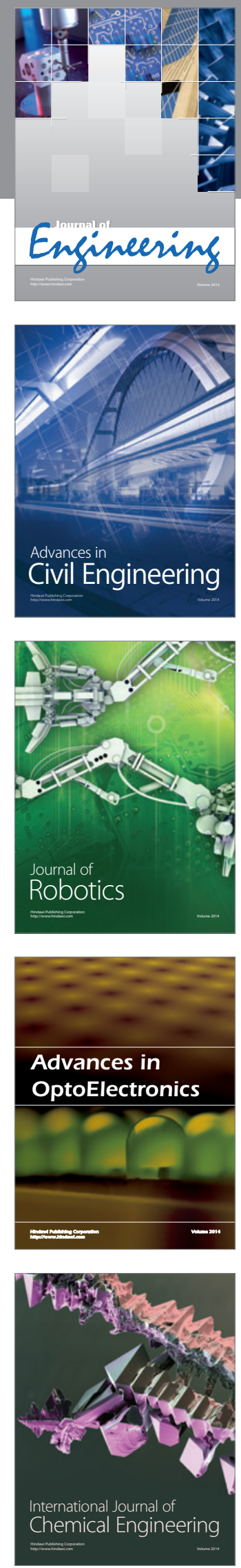

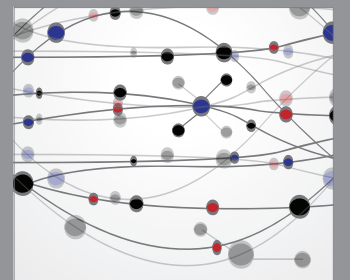

The Scientific World Journal
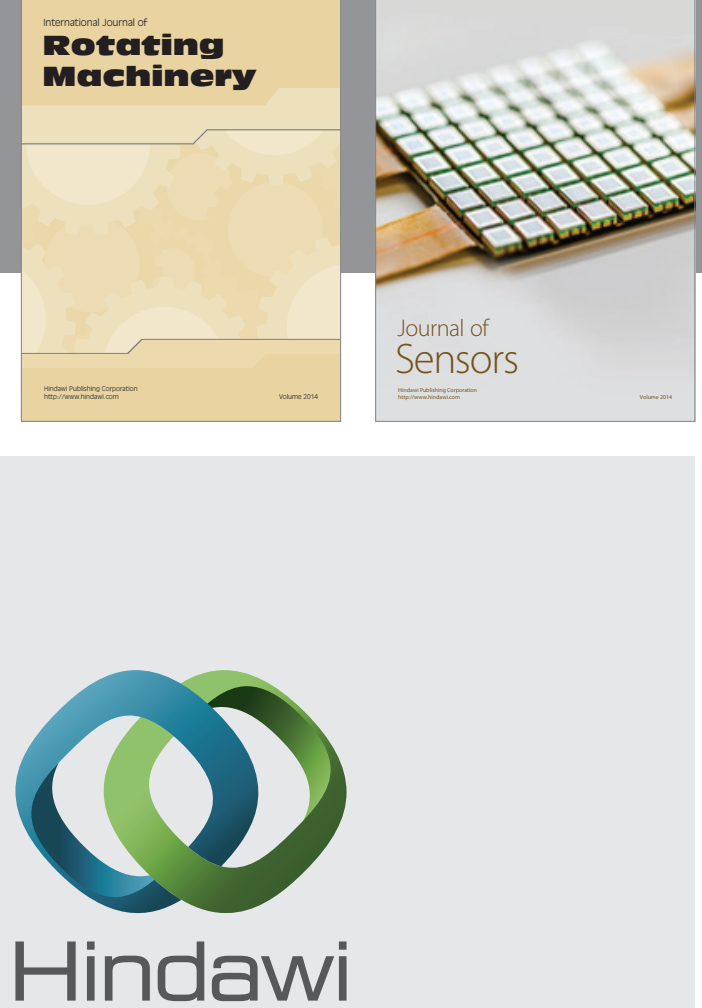

Submit your manuscripts at http://www.hindawi.com
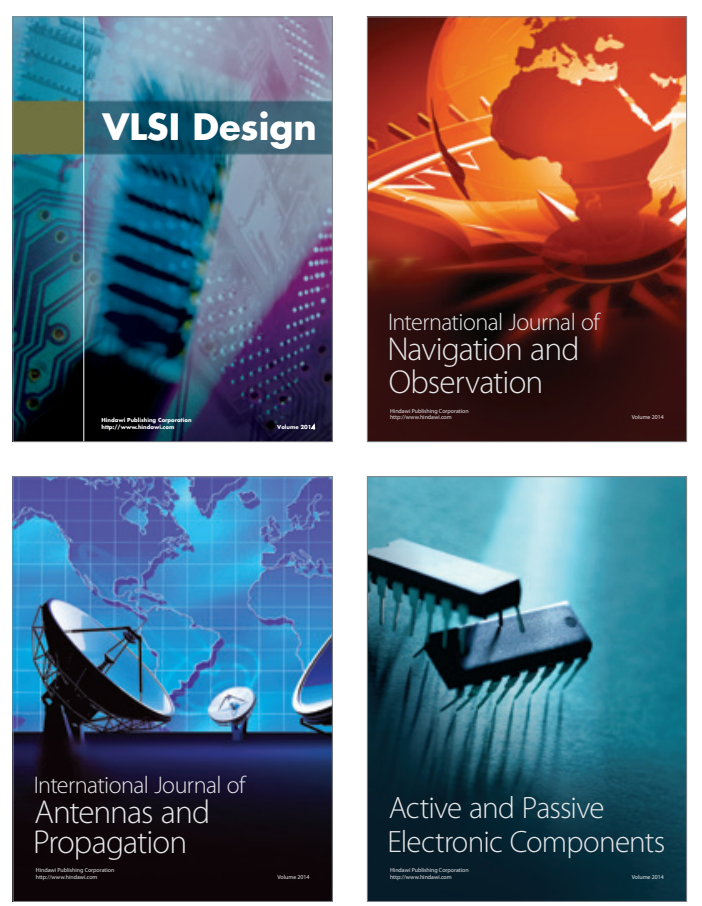
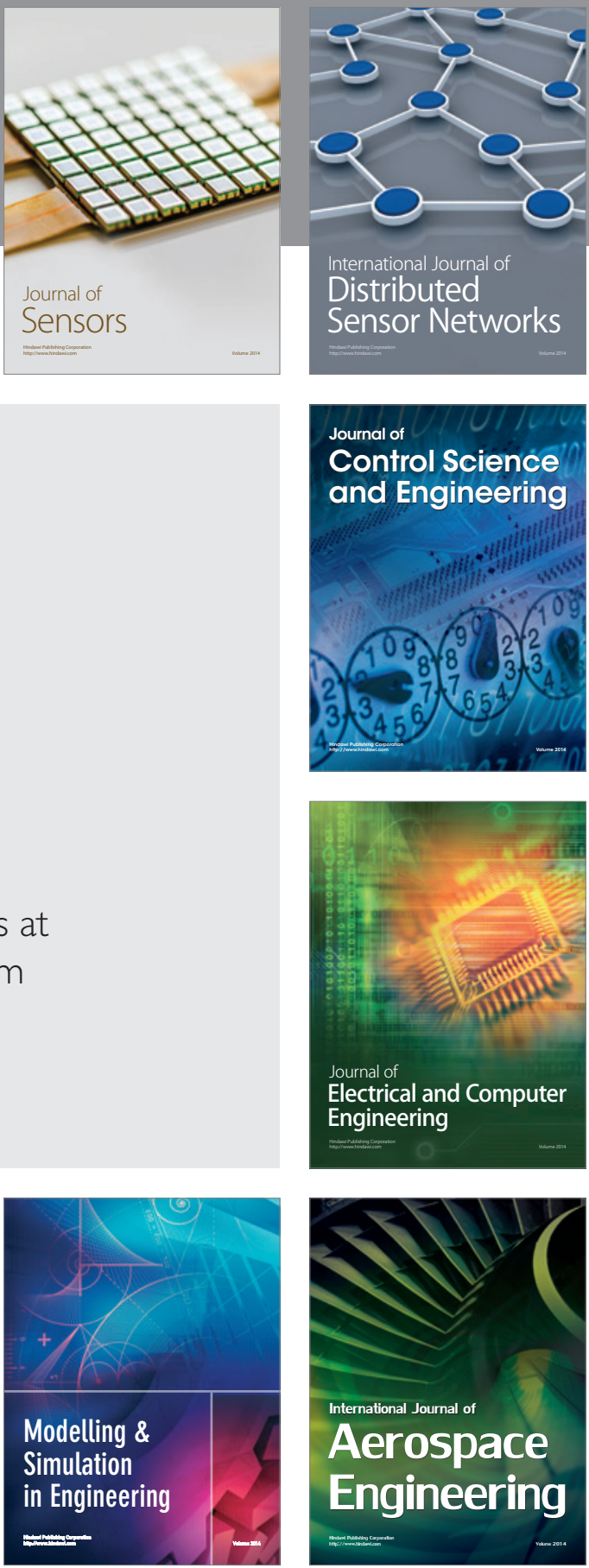

Journal of

Control Science

and Engineering
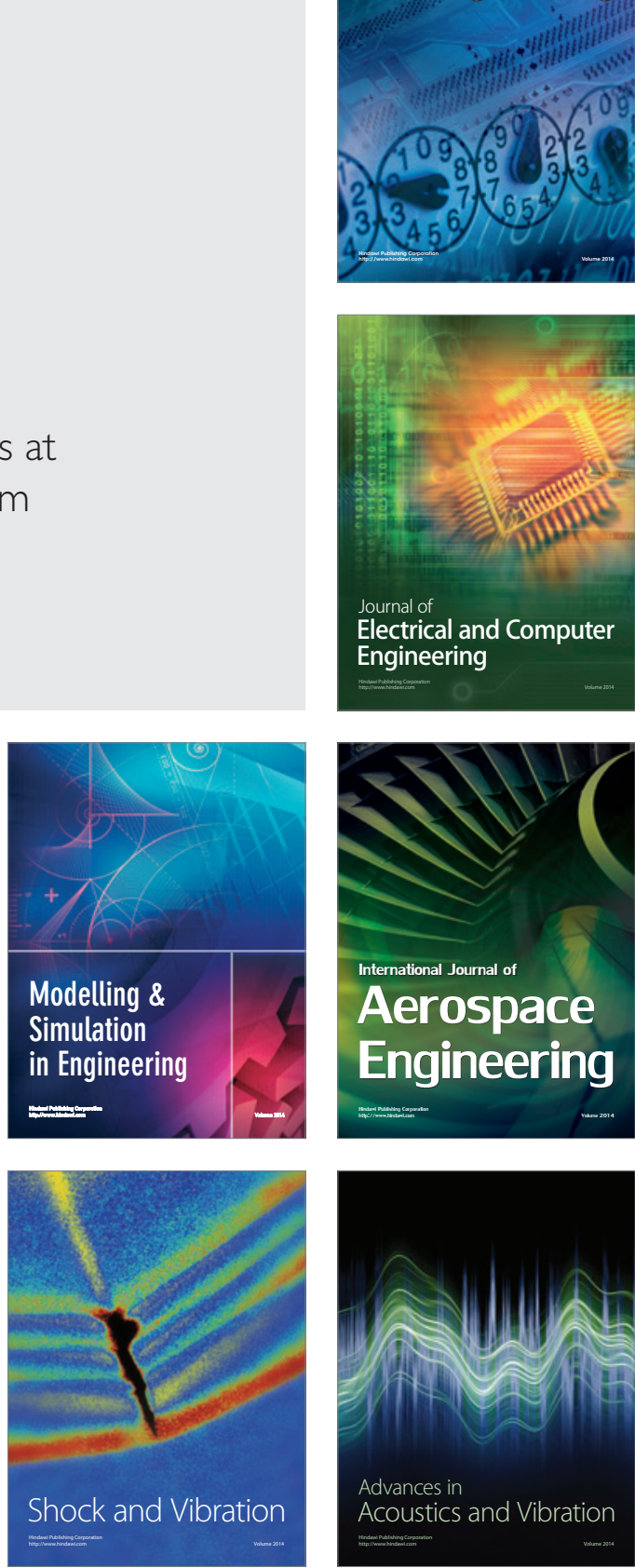